\title{
Transcriptional knockout of steroidogenic factor 1 in vivo in Oreochromis niloticus increased weight and suppressed gonad development using antisense RNA
}

\section{Zhe Cao}

Chinese Academy of Fishery Sciences Freshwater Fisheries Research Center Jun Qiang ( $\nabla$ qiangj@ffrc.cn )

Chinese Academy of Fishery Sciences Freshwater Fisheries Research Center 0003-4718-6618

\section{Jun Zhu}

Chinese Academy of Fishery Sciences Freshwater Fisheries Research Center Hong Li

Chinese Academy of Fishery Sciences Freshwater Fisheries Research Center Yi Tao

Chinese Academy of Fishery Sciences Freshwater Fisheries Research Center Jie He

Chinese Academy of Fishery Sciences Freshwater Fisheries Research Center pao xu

Chinese Academy of Fishery Sciences Freshwater Fisheries Research Center

\section{Zai Dong}

Chinese Academy of Fishery Sciences Freshwater Fisheries Research Center Jin Bao

Chinese Academy of Fishery Sciences Freshwater Fisheries Research Center Jun Ma

Nanjing Agricultural University

Ming Li

Nanjing Agricultural University

\section{Article}

Keywords: Steroidogenic factor 1 (sf1), Nile tilapia, experimental fish, gene function, biological breeding Posted Date: January 4th, 2021 
DOI: https://doi.org/10.21203/rs.3.rs-138053/v1

License: (c) (1) This work is licensed under a Creative Commons Attribution 4.0 International License. Read Full License 


\section{Transcriptional knockout of steroidogenic factor 1 in vivo in}

\section{Oreochromis niloticus increased weight and suppressed gonad development using antisense RNA}

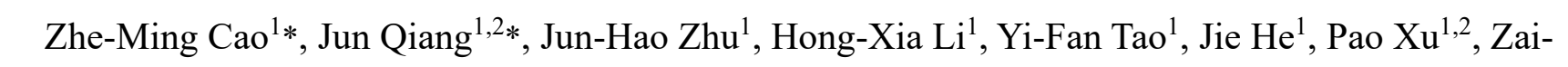
Jie Dong ${ }^{1}$, Jin-Wen Bao ${ }^{1}$, Jun-Lei Ma², Ming-Xiao $\mathrm{Li}^{2}$ 7

1. Key Laboratory of Freshwater Fisheries and Germplasm Resources Utilization, Ministry of Agriculture, Freshwater Fisheries Research Center, Chinese Academy of Fishery Sciences, Wuxi 214081, Jiangsu, China

2. Wuxi Fisheries College, Nanjing Agricultural University, Wuxi 214081, China

*These authors contributed equally to this study

Corresponding authors. Jun Qiang (qiangj@ffrc.cn) and Pao Xu (xup@ffrc.cn) 
15 Steroidogenic factor $1(\mathrm{sfl})$ is an important regulator of gonad development and function in mammals.

16 However, study of sf1 in fish is limited to cloning and expression and in vitro experiments. Using 17 antisense RNA we knockout transcription of the sf1 gene in Nile tilapia Oreochromis niloticus, and 18 obtain experimental fish in vivo. We demonstrate that antisense RNA can silence sf1 transcription and 19 protein expression, and report suppression of sf1 transcription to affect gonad development and 20 external genitalia formation in Nile tilapia. We also report disfunction of retinal metabolism and fatty 21 acid metabolism to be important causes of weight gain and gonad abnormality with sf1 suppression. 22 The feasibility of using antisense RNA for gene editing in fish is verified, and a new way of studying gene function and performing biological breeding is presented. 
Recent rapid developments in gene-editing technologies such as the CRISPR/Cas9 system have enabled efficient and precise biological breeding. Experiments on fishes have been conducted mostly on model species such as zebrafish (Danio rerio) $)^{1,2}$ and medaka (Oryzias latipes) ${ }^{3}$ as opposed to commercially important farmed fishes for two main reasons: 1) eggs of most farmed fishes are small, and an osmotic pressure difference is required when eggs are fertilized; the egg internal pressure is relatively high, and egg yolk is contained within a membrane that is easily broken during microinjection or electroporation, rendering the success rate extremely low and the operation cumbersome. 2) Normally, when a target gene is edited using gene-editing technology (such as CRISPR/Cas9 and zinc finger nuclease technology) in stage I fertilized cells, since the sperm has combined with the egg and fertilization is complete the maternal genetic material begins transcription and translation. Therefore, the $\mathrm{P}_{0}$ generation of experimental fish has a low positive rate and many chimeras. Multiple generations of breeding pairs are required to obtain homozygous individuals with the target gene knocked out, prolonging the experimental period. Many experimental fish for which genes have been knocked out using this editing technology have stagnated at the $\mathrm{P}_{0}$ generation $^{4-6}$. The low specificity of the insertion site and the high off-target rate also limit application of CRISPR/Cas9 and other gene-editing technologies in farmed fish, hampering commercialization ${ }^{7}$.

Steroidogenic factor 1 (sfl) is a member of the superfamily of nuclear receptors, also referred to as Ad4BP or NR5A1. sf1 is a key regulator of estrogen and androgen synthesis. In vertebrates, almost all steroid synthase genes are target genes regulated by $\mathrm{sfl}^{8}$. $\mathrm{sf1}$-deficient mice have abnormal adrenal and gonad development, accompanied by gonadotropin loss and sex reversal (male to female) ${ }^{9}$. Research on sfl in fish has focused mostly on cloning and expression and in vitro cell experiments; sf1 has been reported from medaka ${ }^{10}$ zebrafish $^{11,12}$, Nile tilapia (Oreochromis niloticus) ${ }^{13}$, a gobiid fish (Trimma okinawae) ${ }^{14}$, channel catfish (Ictalurus punctatus) ${ }^{15}$, black porgy (Acanthopagrus schlegelii) ${ }^{16}$, and the air-breathing catfish (Clarias gariepinus $)^{17}$. It is also widely expressed in the brain, hypothalamus, pituitary, head kidney, liver, testis, and ovary tissues of Arctic char (Salvelinus alpinus $)^{18}$ and zebrafish ${ }^{19}$. sf1 is mainly expressed in steroid-producing cells in the early stages of gonad development, and prophase and yolk stage cells ${ }^{20,21}$. The lack of reports of sf1 in bony fish body function can largely be attributed to technical reasons. Using CRISPR/Cas9 technology, Wang's team first studied the mutant Nile tilapia sfl and analyzed gonadal phenotype and $\mathrm{P}_{0}$ mutant gene expression. Preliminary analysis suggests that sfl loss in gonad development may cause females to transition to males ${ }^{22}$. 
Antisense RNA is a single stranded RNA that is complementary to a protein coding messenger RNA (mRNA) with which it hybridizes, and thereby blocks its translation into protein. By interfering with mRNA translation and post-transport processing of related genes, antisense RNA causes loss of target gene function. Tomizawa et $a .^{23}$ first used antisense RNA technology to inhibit the enterobactin-producing Col E1 plasmid of Escherichia coli. This technology has been widely used in plants: Oeller et al. ${ }^{24}$ reported the cauliflower mosaic virus (CaMV) 35S promoter and nopaline synthase (NOS) terminator changed the rate-limiting enzyme in the ethylene biosynthesis pathwaysynthesis of 1-aminocyclopropanecarboxylic acid, which can inhibit tomato fruit ripening. This technology is mostly used to improve breeding of plant traits ${ }^{25,26}$. Experiments on animals are limited to cell-based assays ${ }^{27,28}$, with no reports of live animals being produced using antisense RNA technology.

Nile tilapia (Oreochromis niloticus) is an extensively cultured freshwater fish that is farmed in more than 100 countries and regions. It grows fast, reproduces well, and male and female growth obviously differs. Selection of tilapia as a research subject has universal application. Based on the principle of antisense RNA, we pioneered a set of efficient RNA function knockout technology. We first designed an antisense RNA fragment based on the Nile tilapia sfl gene, designing primers, then constructing, cloning and amplifying the target fragment. We then transfected the target fragment into an egg through a fertilization hole, and added sperm to complete fertilization [technology patents have been applied for in the United States (nos 17/030, 023) and Germany (no 102020126733.9)]. We used this technology to successfully culture three batches of tilapia lacking the sf1 function between 2019 and 2020. The rate of insertion of the target fragment into gonad tissue exceeded $80 \%$, with a good knockout effect on the transcription of sf1. This work mainly reports and analyzes related experimental results.

We report changes in gonad characteristics and tissue structure of Nile tilapia. Use qPCR and western blot techniques we identify the level of translational knockout in vivo. Transcriptome and proteomics techniques are used to co-analyze changes in downstream genes and functions caused by sf1 loss. We overcome obstacles in traditional gene editing through introduction of foreign fragments and target site selection, and the $\mathrm{P}_{0}$ generation obtains stable trait performance. Use of this geneediting technology provides a new experimental means to perform biological breeding and target gene research, with strong commercialization prospects. We also provide theoretical support for studying the regulatory mechanism of sfl, which may further overcome obstacles in treatment of sfl deficiency 
diseases in humans.

\section{Results}

Analysis of positive rate of experimental fish in gonad tissue. To identify the efficiency of antisense RNA introduction, we tested gonad tissues of 17 experimental fishes cultured for $80 \mathrm{~d}$. The target plasmid (about $1000 \mathrm{bp}$ ) containing antisense RNA fragments (Fig. 1a, A1-7 and E1-10) was amplified from gonad tissues of 15 fish, with a positive rate of $88.2 \%$. The negative control (NC) group had a target plasmid band of about $900 \mathrm{bp}$ (B1-7 and D1-4). The control group had no band at the position of 900-1000 bp (E1-4). Each sample contained four transfected antisense RNA sequences.

Antisense RNA inhibits expression of sf1 mRNA and protein in fish gonad tissue. To analyze the inhibitory effect of antisense RNA on the target gene, we used qRT-PCR and western blot (WB) to detect transcription and protein expression levels in the gonadal sfl gene. Expression levels of sfl mRNA and the transcript variant X1 mRNA in testis tissue of male fish in the experimental group were (extremely) significantly lower than those in the control and NC groups $(P<0.01)$ (Fig. 1b). Expression levels of sf1 mRNA and the transcript variant X1 mRNA in ovarian tissues of female fish in the experimental group were also significantly lower than those in the control and NC groups (Fig. $1 \mathrm{c}, P<0.05)$. The molecular weight of sf1 protein synthesized in this experiment ranged $55-65 \mathrm{kDa}$. Obvious protein bands of molecular weight 55-70 kDa occurred (Figs. 1c), indicating the synthesized protein was effective. The expression level of sf1 protein in male (Fig. 1d: b1-4) and female (Fig. 1e: d1-4) fish in the experimental group was significantly lower than in the control (male a1 and a2, and female $\mathrm{c} 1$ and $\mathrm{c} 2$ ) and $\mathrm{NC}$ (male $\mathrm{a} 3$ and $\mathrm{a} 4$, and female $\mathrm{c} 3$ and $\mathrm{c} 4$ ) groups. The introduced antisense RNA fragment effectively suppressed the transcriptional expression and protein level of the sf1 gene in gonad tissues, and achieved transcription knockout of the sf1 gene.

\section{Figure 1 inserted here}

Knocking out sf1 transcription affects formation of external reproductive organs. To observe changes in fish reproductive organs after sf1 transcription knockout, images of experimental tilapia were taken. Male and female tilapia in the transcription knockout group were more round (Fig. 2a and $\mathrm{b}$ ), and their body weights were significantly higher than control and NC group fishes (Table 1, $P<0.05)$. After $180 \mathrm{~d}$ culture, control group male and female fish reached sexual maturity, and their genitals were obviously red and convex (Fig. 2a and b). The anus and urogenital opening were clearly 
visible in males; the urogenital openings had a small cylindrical white protruding tip (Fig. 2a), and a small amount of semen extruded when the abdomen was gently squeezed. Male fish in the sfl transcription knockout group (M-sf1') had a larger anus and a slightly convex white organ (Fig. 2a), and semen did not extrude when the abdomen was gently squeezed. Control group female fish had an obvious anus and genital and urinary openings; when the abdomen was gently squeezed (Fig. 2b), some individuals discharged eggs. Females in the sfl transcription knockout group (F-sf1') had a large anus and slightly convex red organ (Fig. 2b), and their genital and urinary openings could not be distinguished.

\section{Knockout of sf1 transcription interfered with development and maturation of testis and ovary}

tissues. To examine the effect of sf1 transcription knockout on development of gonad tissue, gonads were weighed and tissues were sectioned. Control and NC group male gonad weights were $2.36 \mathrm{~g}$ and $2.41 \mathrm{~g}$, respectively, with GSI values of 0.975 and 0.969 - significantly higher than $0.51 \mathrm{~g}$ and 0.157 of transcription knockout group fish (Table 1). Control group testes were thick and long; testes of knockout group fish were thinner (Fig. 2a). After HE staining, control and NC group testis appeared to comprise many seminiferous tubules and intertubular structures, with sperm (Sp) evenly distributed in the lumen center and variably sized seminiferous tubules (Fig. 2c). Each seminiferous tubule contained spermatogenic vesicles at different developmental stages, including spermatogonia $(\mathrm{Sg})$, primary spermatocytes (PS), second spermatocytes (SS), and spermatocytes (St). However, the testis of males in the sf1 knockout group (M-sf1', Fig. 2c) had decreased numbers of seminiferous vesicles in the spermatogenic tubules, and Sg, PS, SS and St. Additionally, some sperm gathered in the lumen of the seminiferous tubules, severe vacuolation occurred within them, and the voids in the lumen increased significantly.

Control and NC group female gonad weights and GSI values (5.30 g, 2.539; $5.48 \mathrm{~g}, 2.403$; respectively), were significantly higher than values for experimental group fish (3.21g and 1.073). In control and NC group ovarian tissues (Fig. 2c), tightly packed oocytes at various (II-V) stages were clearly visible, with elliptical mature stage $\mathrm{V}$ oocytes full of large yolk particles. However, ovarian development in the sf1 transcription knockout fishes (F-sf1-, Fig. 2c) was more complicated, with gonads containing many cavities with relatively scattered, low numbers of oocytes of stages II-IV (mainly II). Some follicles are atresia, and oocyte membrane folding occurs.

Knockout of sf1 transcription affects serum hormone levels. To identify the effect of sf1 transcription knockout on serum hormones, we determined serum T, GnRH, LH, FSH and $\mathrm{E}_{2}$ levels 
in male (Fig. 2d) and female (Fig. 2e) fish after $180 \mathrm{~d}$ culture. In males, sf1 transcription knockout fish (M-sf1') had significantly lower serum T than control group fish; however, serum GnRH, LH, FSH and $\mathrm{E}_{2}$ levels were significantly higher than in control group fish $(P<0.05)$. There was no significant difference between control and NC groups $(P>0.05)$. In females, serum LH and $\mathrm{E}_{2}$ levels

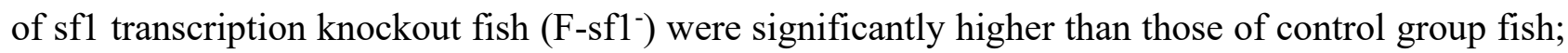
however, the serum $\mathrm{T}$ and GnRH contents were significantly lower than control group fishes $(P<0.05)$. There was no significant difference in serum FSH content in females among experimental groups $(P>0.05)$.

Figure 2 inserted here

Knockout of sf1 transcription regulates mRNA profiles in gonad tissue genes. We analyzed changes in transcription levels of sfl transcription knockout and control group fish gonad tissues. Based on Nile tilapia genome information, we performed comparison and quality identification of transcriptome sequencing results. In males, we sequenced 22,283 genes; 1675 differentially expressed (DE) genes were found between the sf1 transcription knockout and control groups (Fig. 3a), of which 509 were up-regulated and 1166 were down-regulated. In females, 22,283 genes were sequenced; 1726 DEGs were found in the sf1 transcription knockout and control (Fig. 3b) groups, of which 690 were up-regulated and 1036 were down-regulated.

Knockout of sf1 transcription regulates protein profiles in gonad tissue genes. For males, 8232 proteins and 7557 quantitative proteins were identified (Fig. 3a); 469 differentially abundant (DA) proteins were found in the sfl transcription knockout and control groups, of which 96 were upregulated and 377 were down-regulated. For females, 6024 proteins and 5534 quantitative proteins were sequenced (Fig. 3b); 636 DA proteins were found in the sf1 transcription knockout and control groups, of which 242 were up-regulated and 394 were down-regulated.

\section{Combined transcriptome-proteome analysis of the effects of sf1 transcription knockout on} gonad development and metabolism. Based on transcriptome data, we conducted a regulated analysis for DE gene-DA protein pairs. The 44 and 74 DE genes in male (Fig. 3c) and female (Fig. 3d) fish showed significant differences from common proteins by integrated analysis of transcriptome and proteome sequencing results, with the criteria for DE gene being $P$ value $\leq 0.05$, fold change (FC) $\geq 2$ or $\mathrm{FC} \leq 0.5$, and for the DA protein $P$ value $\leq 0.05, \mathrm{FC} \geq 1.2$ or $\mathrm{FC} \leq 0.833$. For males, $18 \mathrm{DE}$ genes and 16 DA proteins were up-regulated (Fig. 3c and e); 13 genes and common proteins were upregulated; $34 \mathrm{DE}$ genes and $36 \mathrm{DA}$ proteins were down-regulated, of which $31 \mathrm{DE}$ genes and common 
proteins were down-regulated. For females (Fig. 3d and f), 27 DE genes and 34 DA proteins were upregulated, of which $23 \mathrm{DE}$ genes and common proteins were up-regulated; $62 \mathrm{DE}$ genes and $55 \mathrm{DA}$ proteins were down-regulated, with $51 \mathrm{DE}$ genes and common proteins down-regulated.

Heatmaps of DE gene-DA protein pairs for both sf1 transcription knockout and control groups are shown in Fig. 3g (male) and Fig. 3h (female). Overall expression patterns of DE genes and common DA proteins in the sfl transcription knockout group differ from those in the control group. In the sfl transcription knockout group, most genes and proteins in male fish and female fish were down-regulated (blue band).

Figure 3 inserted here

We used GO and KEGG to analyze the function, class, and enrichment pathways of selected DE gene-DA protein pairs. Compared with the control group, the GO annotation of males in the sfl knockout group involved 50 functional groups, including 25 biological processes, 15 cellular components, and 10 molecular functions (Figs. 2a). Of biological processes, cell adhesion, xenobiotic metabolic, and bile acid biosynthetic processes predominated; of cellular components, those integral to the membrane and cytosol predominated; of those with a molecular function, those involved with ATP, metal ion and protein binding predominated. GO annotation of females involved 47 functional groups, comprising 24 biological processes, 15 cellular components, and 8 molecular functions (Figs. b). Of biological processes, those involved with proteolysis and cell adhesion predominated; of cellular components, those involved with membrane, extracellular regions, and the cytoplasm predominated; of those with a molecular function, those involved with metal ion and ATP binding predominated.

For KEGG (Fig. 4a) the main class of related DE gene-DA protein pairs in male mainly included metabolism and the organismal system; a large number of DE gene-DA protein pairs were enriched in the KEGG pathways of cell adhesion molecules (CAMs), steroid hormone biosynthesis, arachidonic acid metabolism, apoptosis, pentose and glucuronate interconversions, and aldosteroneregulated sodium reabsorption. Related DE gene-DA protein pairs in females also predominantly related to the metabolism and organismal systems (Fig. 4b); a large number of DE gene-DA protein pairs were enriched in the KEGG pathways of lysosome, extracellular matrix (ECM)-receptor interaction, drug (other enzyme) and retinol metabolism, xenobiotic metabolism by cytochrome P450, and steroid hormone biosynthesis.

We used Cytoscape V3.7.1 to construct a DE-gene network map based on related DE gene-DE 
protein pairs. Only 4 of 44 genes were involved in the regulatory network of male fish, with fewer associations between genes (Figs. 3a). For females, 48 of 74 genes were involved in the regulatory network, of which ephx2, col1a1, col1a2, ctsd, ugt, aldh1a2, and fn1b are closely related to other DE genes (Figs. 3b). Of these, colla1 is linked to 7 genes, colla 2 and ctsd to 8 genes, and fn $1 \mathrm{~b}$ to 5 genes. Therefore, these DE genes are closely connected with other related DE gene-DE protein pairs, and may be involved in regulation of female growth and development after sf1 transcription knockout.

To verify correlations between transcriptional and protein levels, we mainly selected the topranked KEGG pathways for correlation analysis. Of these, gene and protein expression in the steroid hormone biosynthesis pathway of males are positively correlated (Figs. 4a); genes and proteins of ECM-receptor interaction, retinol metabolism, drug metabolism-cytochrome P450, metabolism of xenobiotics by cytochrome P450, and steroid hormone biosynthesis pathways in females are all positively correlated (Figs. 4b). We used qRT-PCR to further verify the accuracy of some gene sequencing results ( 7 genes in both males and females). Transcription and protein levels of hsd11b2, ephx2, cyp4f3 and fndc 1 in males in the sf1 transcription knockout group ( $\mathrm{sfl}^{-}$) were significantly up-regulated, while capn12, atp1a3b and ugt5a1 gene transcription and protein levels were significantly down-regulated (Fig. 4c). For females, colla2, colla1, fn1b and aldh1a2 gene transcription and protein levels in the sfl transcription knockout group were significantly upregulated, while ctsd, ugtlab and cyp3a65 gene transcription and protein levels were significantly down-regulated (Fig. 4d). Verification results of transcription levels of the 14 selected genes are consistent with transcriptome sequencing results (square of Pearson's correlation coefficient $\left(R^{2}\right)>$ 0.9), indicating that the experimental transcriptome sequencing results are reliable.

\section{Figure 4 inserted here}

\section{Discussion}

We are the first to report sf1 transcription knockout in tilapia using high-efficiency RNA knockout technology. sf1 mRNA and protein expression levels were significantly suppressed. sfl knockout experimental fish manifested obvious defects in gonad and external genitalia development when they should have been sexually mature, and their body weight differed from those fishes in the control group. Our study of fish gene function by knockout gene transcription has overcome traditional obstacles with gene editing technologies, and problems associated with chimeras and workload. Our technique has considerable commercial potential. sfl occurs widely in mammals, non-mammalian vertebrates, and invertebrates, and is a highly conserved gene ${ }^{29}$. Mice in which sfl has been knocked 
out have a significant weight gain advantage ${ }^{30}$. The pituitary-specifically knocked-out sfl mice significantly reduced the level of pituitary gonadotropin, manifesting severe gonad and external genitalia hypoplasia, a significant reduction in the number of germ cells, and a lack of mature sperm being formed ${ }^{31}$. Although the expression and function of sf1 have been studied in detail in mammals, there has been limited study of sf1 in non-mammals, especially in bony fishes. For this reason, it has been rarely reported in studies of bony fish gonad development.

sf1 transcription knockout reduces the GSI of tilapia and increases body weight. In bony fish a change in GSI is related to maturity stage, and it represents an important indicator of the spawning cycle. We report the weight of male and female fish in the sf1 transcription knockout group to be 1.47 $\times$ and $1.34 \times$ greater than that of control group fish, but for the gonad weight and GSI to be $0.22 \times$ and $0.15 \times($ male $)$ and $0.61 \times$ and $0.45 \times($ female $)$ that of control group fish, respectively. The weight of sf1 knockout gonad-development-deficient mice at 8 weeks age is $2 \times$ the weight of control mice. Obesity is caused mainly by reduced mobility and increased food intake ${ }^{30}$. Experimental fish with sf1 transcription knockout showed significant gonad weight loss and weight gain, which may also be related to a reduction in reproductive energy expenditure.

sf1 transcription knockout causes abnormal secretion of sex hormones, causing defects in gonad and genitalia development. In sfl transcription knockout fish, the serum $\mathrm{T}$ content of males was strongly downregulated. After male mammals enter puberty, testicular function gradually develops, T secretion increases, spermatogonia divide and differentiate, spermatogenesis is initiated, and mature sperm are produced ${ }^{32}$. sfl coordinately regulates the steroid hydroxylase gene and affects the reproductive axis as an integral mediator of steroid production ${ }^{33}$. Like mammals, the fish sfl gene also plays an important role in reproductive regulation of the hypothalamic-pituitary-gonad axis, and is strongly expressed in the pituitary and gonads of common carp (Cyprinus carpio) $)^{34}$. The 5 ' end of some testosterone-producing enzyme genes has an sfl binding site upstream of the transcription start point, suggesting that sf1 may be involved in transcriptional regulation of testosterone-producing enzymes, affecting testosterone synthesis ${ }^{35}$. Therefore, the transcriptional knockout of male fish sf1 may reduce secretion of testosterone during the reproductive period, causing suppression of gonad development, introducing barriers to formation of spermatogonia and spermatocytes, leading to an inability to produce mature sperm. We also report sfl transcription knockout to affect the differentiation and formation of external genitalia, resulting in shorter external genitalia, similar to an sf1 gene abnormality causing penis shortness in children ${ }^{36}$. 
$\mathrm{T}$ is present in the blood of many female teleost fish. As a precursor of $\mathrm{E}_{2}$ synthesis, $\mathrm{T}$ can be used for aromatization in the ovary ${ }^{37}$. T content is related to oocyte maturation, embryonic development and miscarriage rate in women with polycystic ovary syndrome ${ }^{38}$. In the flounder Pseudopleuronectes americanus $\mathrm{T}$ also plays an important role in oocyte maturation and ovulation in winter ${ }^{39}$. Therefore, a decline in serum $\mathrm{T}$ in female fishes in the sfl transcription knockout group resulted in reduced development of oocytes and increased follicular atresia.

sfl can promote expression of some steroid-producing enzymes which affect the activity of genes related to gonad development, including GnRH receptor ${ }^{41}$ and $\mathrm{LH} \beta$-submit ${ }^{41}$. sf1 knockout mice cannot regulate expression of LH $\beta$-subunit, FSH $\beta$-subunit and $\mathrm{GnRH}$ receptor genes ${ }^{42}$. Brown and Mcneilly ${ }^{43}$ reported sheep luteal and follicular estrous cycle phases and sf1 mRNA levels to positively correlate with expression of LH $\beta$-subunit. We report serum $\mathrm{LH}$ and $\mathrm{E}_{2}$ contents of male and female sfl transcription knockout fishes to be significantly higher than those of control group fish during the reproductive period. Our results again confirm that two different sex determination and differentiation systems exist between bony fish and mammals. Changes in sex steroids, temperature and light intensity may promote sex conversion ${ }^{44}$. Different potential ligands dependent on sfl may dynamically exchange when sfl is lacking, activating its transcription pathway to enhance $\mathrm{LH}$ and $\mathrm{E}_{2}$ activity.

GnRH injection can induce a transition from female to male, or male to female at the stage of sex differentiation. GnRH can induce production of gonadotropins, stimulate FSH and LH to enter the peripheral circulation, and act on ovary and testes tissues to regulate follicle formation, ovulation, and sperm and steroid production ${ }^{45}$. Therefore, up-regulation of GnRH in male sfl transcription knockout fishes may promote increased serum FSH and LH levels and down-regulate androgen, causing abnormal spermatocyte and sperm development ${ }^{46}$. However, down-regulation of GnRH in sf1 knockout females may be related to suppression of egg development.

sf1 transcription knockout changes regulatory pathways in male and female gonads. We used transcriptomics and proteomics to study gene and protein expression data. The Illumina platform and Q Exactive plus mass spectrometer were used to identify 12 transcriptome and 12 proteome libraries from the sf1 transcription knockout and control group fishes. For the first time, 44 DE gene-DA protein pairs were identified in male fish, and 74 in female fish.

Clustering and KEGG pathway enrichment analyses revealed many DE genes to be enriched in retinol metabolism and steroid hormone biosynthesis pathways. Combined with abnormal gonad 
tissues, we suggest that the retinol signaling pathway plays an important role in fish reproductive development. Deficiency or excess of retinol can cause testicular lesions and spermatogenesis in male animals ${ }^{47,48}$. Also, in female animals, retinol and its active derivatives can affect ovarian steroid hormone production, oocyte maturation, and early embryonic development ${ }^{49}$. We report a similar phenomenon, with changes in genes in the retinol metabolism pathway possibly affecting spermatogenesis and oocyte development. Uridine diphosphate glucuronyl transferase (UGT) is an important phase II metabolic enzyme. UGT protein can be regulated by a variety of ligand-dependent or independent transcription factors, including the hepatocyte nuclear factor family and nuclear receptor superfamily (constitutive androstane receptor CAR, and pregnane $\mathrm{X}$ receptor PXR). PXR and CAR can form heterodimers with retinoid $\mathrm{X}$ receptors to initiate metabolism of various exogenous or endogenous substances such as hydroxysterol, bile acid, and androgen ${ }^{50}$. Currently, the Ugt5 family is known only from fish and amphibians ${ }^{51}$. The transcriptional expression pattern is sexrelated in Ugt1a and Ugt5a of zebrafish, with Ugtla and Ugt5a1 expression in male gonads significantly higher than in females ${ }^{52}$. We report transcription and protein levels of ugt5a1 in male and ugtlab in female Nile tilapia in the sfl transcription knockout group to be significantly downregulated. sfl knockout may affect retinol metabolism of fish. Retinol and its metabolic derivatives cannot fully bind to the corresponding ligands, causing down-regulation of transcription levels of ugt5a1 and ugt1ab, leading to insufficient synthesis of steroid and sex hormones.

Retinoic acid (RA) is an important biologically active form of vitamin A. It can be oxidized to retinal by retinol dehydrogenase, and then oxidized to all-trans-RA and 9-cis-RA by retinal dehydrogenase. The all-trans-RA is eliminated by CYP1A and CYP3A catabolism, and these synthesis and metabolism processes are regulated by enzymes. When RA synthesis and decomposition disorder occurs, it affects normal body physiological functions, and congenital malformations form more easily, manifested in bone, eye and heart structural abnormalities in zebrafish $^{53,54}$. We also report sf1 transcription knockout female and male Nile tilapia to have abnormally developed external genitalia, suggesting that changes in the retinol metabolism signaling pathway may be involved in development of fish external genitalia. Early oocyte clustering appeared in the sfl transcription knockout group, and the proliferation efficiency of early oocytes may be enhanced. During oogenesis, RA regulates the initiation of meiosis; levels of RA are closely related to aldh1 $\alpha 2$ (RA synthase) and cyp3A65 (RA degrading enzyme). Studies have shown that insufficient levels of aldh1a2 in fish (which causes a decrease in RA levels) can delay the time it takes for cells 
to enter meiosis, while a decrease in cyp3A65 levels (which causes an increase in RA levels) can cause cells to enter meiosis early ${ }^{56,57}$. We report mRNA and protein expression levels of aldh1a2 in female sf1 transcription knockout fish to be significantly up-regulated, cyp3A65 expression levels to be significantly down-regulated, and the ratio of aldh1a2 to cyp3A65 to be significantly up-regulated, indicating that RA levels in experimental group fish may rise, resulting in more oocytes entering the meiotic process earlier. Combined with changes in serum $\mathrm{T}$ and $\mathrm{GnRH}$ levels (although oocytes in sf1 transcription knockout females entered meiosis early), the lack of hormones promoting follicle formation and maturation, and secretion disorders may inhibit further oocyte development, increasing follicular atresia.

As a typical environmental sex determining animal, cortisol plays an important role in the responses of fish to external environmental pressures ${ }^{55}$. Environmental changes significantly increase levels of cortisol in the body or cells, affecting reproductive growth and sex differentiation in fish ${ }^{58,59}$. Hydroxysteroid 11-beta dehydrogenase (Hsd11b) belongs to a family of oxidoreductases, which regulate mutual conversion between active cortisol and inactive corticosterone, and also participate in the physiological process of steroid metabolism ${ }^{60}$. hsd11b2 can reduce the level of cortisol in fish tissues, thereby protecting them from cortisol damage; it is also involved in androgen synthesis ${ }^{61,62}$. We report male fish in the sf1 transcription knockout group to be involved in the regulation of steroid hormone biosynthesis pathway, significantly up-regulates hsd11b2 expression may increase the conversion of active cortisol to inactive $\operatorname{cortisol}^{63}$, then active the synthesis of androgens. During high temperature-induced male transfer in the silverside Odontesthes bonariensis, cortisol promotes androgen production by regulating high expression of hsd11b2, driving testis development ${ }^{64}$. However, GnRH, LH, FSH, and $\mathrm{E}_{2}$ serum levels in male sfl transcription knockout fish were significantly higher than control group fish. Inhibition of sfl expression may promote the conversion of androgens to estrogen, reducing the effect of male fish androgen, explaining why our male fish gonad tissue had more cavities, and decreased sperm count and gonad weight.

Fndc1 has a prominent role in regulating proliferation, apoptosis and migration of prostate cancer in men ${ }^{65}$. Loss of Fndc1 expression causes a loss in fibronectin expression, suppressing androgen receptor expression ${ }^{66}$. Another important feature of fish for which sf1 transcription has been knocked out is their significant increase in body weight. Androgens play an extremely important role in fat metabolism ${ }^{67}$. Testosterone concentrations in viscerally obese men and those with related metabolic diseases are lower than normal, which induces atherosclerosis, dyslipidemia and insulin resistance ${ }^{68}$. 
We report the expression of Fndc1 and serum $\mathrm{T}$ in male fishes in the sfl transcription knockout group to be significantly down-regulated. Testosterone and its active substances can bind to androgen receptors to activate nuclear gene expression, which is considered to be the main way for androgens to perform their functions ${ }^{64,66}$. Fish have similar regulatory pathways as mammals. Male fish lacking sf1 inhibited expression of Fndc1, which may reduce the functional effects of testosterone, leading to defects in gonad function and endocrine metabolism disorders.

The size of the adrenal glands of sfl knockout heterozygous mice is reduced, accompanied by damage caused by corticosterone in response to stress ${ }^{69}$. We report the atpla $3 \mathrm{~b}$ gene in male fishes in the sfl transcription knockout group to be significantly down-regulated. The atpla3 subtype is mainly expressed in the nervous tissue in mammals, the down-regulation of atp $1 \mathrm{a} 3 \mathrm{~b}$ may be related to the lack of sfl causing kidney damage ${ }^{70}$. Meanwhile, the lack of sfl may affect the secretion of aldosterone, thereby affecting the carbohydrate, lipid metabolism and obesity of fish, leading to fish metabolic syndrome.

Cathepsin D, a member of the cathepsin superfamily, is a lysosomal aspartic protease which plays an important role in maintaining tissue homeostasis and metabolism. Cathepsin D inhibitors can protect rat cardiomyocytes from apoptosis induced by free radicals ${ }^{71}$ and inhibit the release of Cyt-c and caspase activation in human fibroblasts, thereby inhibiting the occurrence of apoptosis ${ }^{72}$. The calpain system plays an important role in maintaining the balance of protein metabolism in the body. Abnormal or imbalanced activation of calpain is often associated with pathological conditions ${ }^{73,74}$. In the sf1 transcription knockout group, the activities of calpain 12 in male and cathepsin D in female fish were significantly down-regulated, indicating a change in tissue homeostasis or metabolic function. The specific regulation mechanism needs further analysis. Fish muscle toughness is closely related to collagen content. Studies on gilthead sea bream (Sparus aurata) have shown that increased collagen content increases muscle mechanical strength and cohesion ${ }^{75}$. Both colla1 and colla2 in female fish in the sfl transcription knockout group were significantly up-regulated, which may be related to changes in muscle tissue structure caused by obesity.

Polyunsaturated fatty acids in fat and their derivatives, arachidic acid, have physiological functions such as regulating steroid hormone synthesis and lipid metabolism during fish gonad development ${ }^{76}$. Cholesterol is catalyzed into testosterone with strong activity under the action of steroidogenic acute regulatory protein, $17 \beta$ hydroxylase, and hsd11b2. Testosterone can be catalyzed by aromatase to form estradiol, and participates in female-related physiological metabolism ${ }^{77}$. The 
expression of hsd11b2 in males in the sf1 transcription knockout group was up-regulated, which may increase testosterone synthesis. However, serum testosterone levels in male fish in the knockout group was significantly lower, and the serum estradiol level was significantly higher than that of control fish. Combining the sequencing results, cyp4f3 in the arachidonic acid metabolism pathway of male fish was significantly up-regulated. Whether up-regulation of cyp4f3 can help catalyze the conversion of testosterone to estradiol remains to be verified.

Epoxide hydrolase in mammals is a homodimer enzyme that integrates $\mathrm{N}$-terminal phosphatase activity and C-terminal hydrolase activity. It participates in various physiological regulation processes and plays an important role in blood pressure control and hormone regulation ${ }^{78,79}$. We report the Ephx2 gene and protein levels in males in the sf1 transcription knockout group to be significantly upregulated. Ephx activity in the liver and kidney of male mice was 55\% and $283 \%$ higher than that of female mice, respectively. Ovariectomy can also increase the activity of Ephx in the liver and kidney of mice ${ }^{80}$. These studies indicate that the regulation of Ephx involves sex hormones. The deletion of Ephx's variable transcript gene Ephx2C at the N-terminus can cause inactivation of the phosphatase region, with the highest expression level in the luteal phase of the estrous cycle, indicating that Ephx $2 \mathrm{C}$ phosphatase may play an important role in hormone regulation ${ }^{81}$. Isoprene phosphate, an intermediate metabolite at the branch point of cholesterol biosynthesis, is the N-terminal universal substrate of Ephx. Therefore, the up-regulated expression of Ephx2 may be involved in sex hormone synthesis of males after sf1 transcription knockout. Male fish lacking sf1 may activate a variety of sex hormone synthesis pathways, mediate the reaction of cholesterol synthesis to sex hormones, and compensate for their lack of male hormones. Our results also found sf1-deficient males retain short external genitalia and gonads with developmental defects.

Fibronectin (Fn) is the main adhesion molecule of ECM. In zebrafish, Fn deposition can be seen around the myocardial precursor and in the midline area between the endoderm and the endocardial precursor $^{82}$. Likewise, antisense morpholino knockdown or gene mutations resulting in the loss of Fn1 can inhibit endothelial invasion and migration of steroid-producing tissues ${ }^{83}$. We report the Fn1b gene transcription and protein expression in females in the sfl transcription knockout group to be significantly up-regulated. Although the loss of sfl caused abnormality in female genitalia, it may accelerate the migration of cells in steroid-producing tissues and promote production of steroid hormones in other tissues, thereby alleviating the imbalanced development and metabolism of the fish body after sf1 knockout. 
Overall, we reveal that knocking out genes at the transcriptional level can produce living organisms in animals, and that this gene editing technique is efficient. We analyzed the role of sfl in the development and formation of fish sexual organs; and proved the response mechanisms of a fish

\section{Methods}

\section{Ethics statement}

The study protocols and design were approved by the Ethics Committee at the Freshwater Fisheries Research Centre of the Chinese Academy of Fishery Sciences (FFRC, Wuxi, China). The fish were maintained in well-aerated water and treated with $200 \mathrm{mg} / \mathrm{L}$ tricaine methanesulfonate (MS-222, Sigma-Aldrich, A5040) for rapid deep anesthesia. The samples were extracted based on the Guide for the Care and Use of Laboratory Animals in China.

Experimental fish. We used GIFT (Genetic Improvement of Farmed Tilapia) Nile tilapia from a self- 


\section{5'-AATAACTGACAAACTATTCTTAACAATGAAATGTGTTTATAGTTTGTTGTTGTGGTTTT}

GTTGCTTTCACCTGATCTGACTCGCTGCTGAGTCTCATCTGGTTC-3.'

The antisense RNA comprised a second intron partial sequence, and a first exon partial sequence. The purpose of this design was to interfere with the post-transcriptional processing of the sf1 mRNA precursor.

2. Design of the first antisense RNA sequence of sf1 transcript variant X1-I (anti-sf1 transcript variant X1-I) (Fig. 5b):

5'-TGCTGTCTATATCGGGGATGCTTTAGGAGCCAGAAGGGCCAATAGAAAGTGTAGTACG ATGATGACCATTCAAGAGCTCAGAGTGAAACTCCTTTCCTTCGC-3.’

The antisense RNA comprised a first intron partial sequence, and a 5'-non-translated sequence of 80 bases. The purpose of this design was to interfere with the post-transcriptional processing of the sf1 transcript variant X1 mRNA precursor and the translation initiation of the mRNA.

Design of the first antisense RNA sequence of sf1 transcript variant X1-II (anti-sf1 transcript variant X1-II):

\section{5'-AGGAAAACGGAGGCACTTACCGTGAGCCTTGTCTCCCAACATTTGAGCAGCAGACA} GCAGGCAGGCAGTGATGATTGTCTGATGATTAAAACCTGGATGTAG-3.'

The antisense RNA comprised a second intron partial sequence, a first exon sequence, and a 5terminal non-translated sequence of 60 bases. The purpose of this design was to interfere with the translation initiation of the sfl transcript variant X1 mRNA precursor.

The four designed antisense RNA sequences were sent to GENEWIZ Biotechnology Co., Ltd. (Suzhou, China) for sequence synthesis.

Figure 5 inserted here

PCR amplification. In the experimental group, the four antisense RNA sequences were cloned into the site between Xho $I$ and $X b a I$ in the pcDNA3.1 expression vector (ThermoFisher, K482001) respectively. The cloning product of each antisense RNA was used as a template for subsequent PCR amplification. A pair of specific primers were designed to amplify the template: F1: TTTTGCGCTGCTTCGCGATGTAC, and the reverse primer R1: TCCCCAATCCTCCCCCTTGCTG. The $50 \mu \mathrm{L}$ reaction system contained $25 \mu \mathrm{L}$ of $2 \times$ Mastermix, $2.5 \mu \mathrm{L}$ of each primer $\mathrm{F} 1$ and $\mathrm{R} 1,18 \mu \mathrm{L}$ of ultrapure water, and $2 \mu \mathrm{L}$ of template. The PCR 
amplification procedure involved pre-denaturing at $95^{\circ} \mathrm{C}$ for $2 \mathrm{~min}, 34$ cycles of denaturing at $95^{\circ} \mathrm{C}$ for $30 \mathrm{~s}$, annealing at $50^{\circ} \mathrm{C}$ for $30 \mathrm{~s}$, and extending at $72^{\circ} \mathrm{C}$ for $2 \mathrm{~min}$, and a final extension at $72^{\circ} \mathrm{C}$ for $5 \mathrm{~min}$. The amplified product contained enhancer, TATA box, CAAT box, and a transcription initiation region, tailed with poly-A. In the negative control group, the blank expression vector was amplified according to the above PCR procedure; the reaction system comprised $50 \mu \mathrm{L}$, containing $25 \mu \mathrm{L}$ of $2 \times$ Mastermix, $2.5 \mu \mathrm{L}$ of each primer F1 and R1, $18 \mu \mathrm{L}$ of ultrapure water, and $2 \mu \mathrm{L}$ of template of the blank vector. Except for antisense RNA fragments, the amplified products of the blank vector included enhancer, TATA box, CAAT box, and the transcription initiation region, tailed with poly-A.

Transfection reagent preparation. The PCR amplified products (PCR amplified products of each group above were mixed in a volume ratio of $1: 1: 1: 1$ ), blank expression vector amplified products (negative control group), or ultrapure water (control group), and lipofectamine 2000 (Thermo Fisher Scientific, 11668027) were mixed at a ratio of 1:5; the mixture was equilibrated at room temperature for $30 \mathrm{~min}$.

Artificial insemination and hatching. A single mature female tilapia with salient, ruddy and slightly open gonads was selected. Water around the genitals was gently wiped off with a dry towel. The abdomen was then gently squeezed to extrude mature eggs, which were placed in 3 clean, dry stainless-steel receptacles (150-200 eggs in each). Experiments were divided into control, negative control (transfected with blank templates), and experimental (transfected with target templates) groups.

Into a liter of water, $6 \mathrm{~g}$ of sodium chloride, $0.1 \mathrm{~g}$ of potassium chloride, $0.1 \mathrm{~g}$ of calcium chloride, $0.1 \mathrm{~g}$ of sodium bicarbonate, $0.1 \mathrm{~g}$ of sodium dihydrogen phosphate, and $1.2 \mathrm{~g}$ of glucose was dissolved. Into each receptacle, $1.5 \mathrm{~mL}$ of this solution was added to promote the opening of fertilization hole, after which $0.8 \mathrm{~mL}$ of transfection reagent was added. Receptacles were gently shaken for 15 min to allow the antisense RNA fragment to enter the eggs through the fertilization hole. For the negative control group, $0.8 \mathrm{~mL}$ of transfection reagent containing blank vector was added. For the control group, $0.8 \mathrm{~mL}$ of transfection reagent containing ultrapure water was added.

One male fish with well-developed gonads (genital pores ruddy and salient) was selected, from which $0.2 \mathrm{~mL}$ of semen was gently sucked and placed into each receptacle containing eggs using a disposable dropper. The mixture of eggs and sperm was then stirred for $30 \mathrm{~s}$ using goose feathers; 2 $\mathrm{mL}$ of incubation water was added to complete the artificial insemination process. 
Two further female fish of the same family (time interval 3-8 days) were selected for replication.

Eggs were manually squeezed as previously, then divided into 3 treatment groups with 120-180 eggs in each. Three separate males of the same family were used in artificial insemination experiments. Fertilized eggs were placed into three hatching jars, with water temperature held at $29^{\circ} \mathrm{C}$, and water flow rate $5 \mathrm{~L} / \mathrm{min}$ to ensure that fertilized eggs rolled fully. Many fish fry hatched after $92 \mathrm{~h}$, with the hatching rate of fertilized eggs exceeding $85 \%$.

Experimental fish farming and management. Newly hatched larvae (50-60) were placed into a 30 L water circulating system for $30 \mathrm{~d}$. Larvae were fed (45.0\% crude protein, $8.0 \%$ fat content) four times daily, $15 \%-20 \%$ their body weight. Larvae (25) were then transferred to each of 4 or $5 \times 1.2$ $\mathrm{m}^{3}$ tanks for culture. Fish were fed (crude protein content $32.0 \%$, fat content $8.0 \%$ ) twice daily, 5\%$10 \%$ their body weight, for $150 \mathrm{~d}$. Dissolved oxygen was maintained above $5 \mathrm{mg} \cdot \mathrm{L}^{-1}, \mathrm{pH}$ at $7.6 \pm 0.2$, and ammonia nitrogen and nitrite below $0.02 \mathrm{mg} \cdot \mathrm{L}^{-1}$ and $0.03 \mathrm{mg} \cdot \mathrm{L}^{-1}$, respectively.

Detection of transfection of antisense RNA fragment. When newly hatched larvae were cultured for $80 \mathrm{~d}$, 5 fish were randomly selected from each tank. Following deep anesthesia with MS-222 solution $(200 \mathrm{mg} / \mathrm{L})$, the gonads of each fish were excised. A MiniBEST Universal Genomic DNA Extraction Kit Ver 5.0 (TakaRa, 9765-1) was used for genomic DNA extraction. The $20 \mu \mathrm{L}$ reaction included $0.5 \mu \mathrm{L}$ of each upstream and downstream primer (F1: TTTTGCGCTGCTTCGCGATGTAC; R1: TCCCAATCCTCCCCCTTGCTG, $10 \mathrm{mmol} / \mu \mathrm{L}$ ), $1 \mu \mathrm{L}$ of cDNA, $10 \mu \mathrm{L}$ of Premix Taq (LA Taq Version 2.0, TAKARA, RR901A), and $8 \mu \mathrm{L}$ of RNase-free water. The reaction program was $94^{\circ} \mathrm{C}$ for $2 \mathrm{~min}$, followed by 35 cycles at $95^{\circ} \mathrm{C}$ for $30 \mathrm{~s}, 50^{\circ} \mathrm{C}$ for $30 \mathrm{~s}$, and $72^{\circ} \mathrm{C}$ for $2 \mathrm{~min}$, and finally $72^{\circ} \mathrm{C}$ for $5 \mathrm{~min}$. We made an agarose gel $(0.3 \mathrm{~g}$ agarose $+30 \mathrm{ml} 1 \times \mathrm{TAE})$ and electrophoresed PCR products for 20 min under $300 \mathrm{v}$. A DNA gel recovery kit (Axygen, AP-GX-50) was used to recover target fragments. Through cloning and transformation, 15-20 monoclonal colonies were selected from each sample and sent to GENEWIZ Biotechnology Co., Ltd. for sequencing.

Sampling. We mainly sampled and analyzed fish from first batch of experiments, using those from second and third batch of experiments as supplements and/or to verify results from the first experiment. Feeding of fish ceased 1 day prior to the end of each experiment. We used MS-222 solution (200 mg/L) for deep anesthesia, selected 6 fish from each tank (24-30 fish in total), and took $1 \mathrm{ml}$ blood from the tail vein of each fish after weighing using a $2.5 \mathrm{ml}$ sterile syringe; blood was stored in $1.5 \mathrm{ml}$ Eppendorf centrifuge tubes. After standing for $2 \mathrm{~h}$ at $4{ }^{\circ} \mathrm{C}$, the sample was centrifuged at $8000 \mathrm{~g} / \mathrm{min}$ for $5 \mathrm{~min}$; the upper serum was separated and stored at $-40^{\circ} \mathrm{C}$ for serum hormone level 
analysis. The whole gland tissue was dissected, weighed and photographed. A further 5 fish were removed from each tank, and after deep anesthesia, their gonad was excised and divided into 4 parts: 3 parts stored in cryopreservation tubes and frozen in liquid nitrogen for omics, gene expression and protein level analysis, and 1 part fixed in $4 \%$ paraformaldehyde for tissue structure analysis.

Gonadosomatic (GSI) index determination. GSI was calculated according to: GSI = [gonad mass (g) / body mass $(\mathrm{g})] \times 100 \%$.

RNA extraction, reverse transcription and quantitative PCR. Total gonad tissue RNA was extracted using a TRIzol kit (Invitrogen, 15596026). cDNA was synthesized with reference to the corresponding PrimeScript RT Master Mix reverse transcription kit instructions (Takara, RR036A); gene expression was detected through SYBR Premix Ex Taq kit operation steps (Takara, RR420). Gene relative expression levels were calculated using $\beta$-action as an internal reference. The expression level of related mRNA genes was detected using an ABI QuantStudio 5 Real-Time PCR System. The primer design is presented in Tables 1 . The $20 \mu \mathrm{L}$ of reaction system contained $0.6 \mu \mathrm{L}$ of each upstream and downstream primer $(10 \mathrm{mmol} / \mu \mathrm{L}), 1 \mu \mathrm{L}$ of cDNA, $10 \mu \mathrm{L}$ of $2 \times$ SYBR Premix Ex Taq II, with sterilized double distilled water added to make up $20 \mu \mathrm{L}$. The reaction program was $95^{\circ} \mathrm{C}$ for $5 \mathrm{~min}$, then 40 cycles at $95^{\circ} \mathrm{C}$ for $15 \mathrm{~s}$ and $60^{\circ} \mathrm{C}$ for $60 \mathrm{~s}$; the dissolution curve program after the reaction was $95^{\circ} \mathrm{C}$ for $15 \mathrm{~s}, 60^{\circ} \mathrm{C}$ for $15 \mathrm{~s}$, and $95^{\circ} \mathrm{C}$ for $15 \mathrm{~s}$. Each reaction was replicated three times, and each test sample contained a negative control without template to eliminate false positive results.

Protein separation, protein quantification and western blot. The protein expression level of target genes in gonad tissue was detected by western blot. A sample of $0.05 \mathrm{~g}$ of gonad tissue was selected and crushed with liquid nitrogen and a mortar; $1 \mathrm{~mL}$ of platelet aggregation buffer induced by ristomycin (containing $1 \% 10 \mathrm{mg} / \mathrm{mL}$ phenylmethanesulfonyl fluoride) was added; the mixture was then homogenated with Polytron (PT2500E, KINEMATICA, Switzerland) homogenizer at $4^{\circ} \mathrm{C}$ and $15,000 \mathrm{~g}$ for $1 \mathrm{~min}$. The protein supernatant was aspirated after being centrifuged at $4^{\circ} \mathrm{C}$ and 12,000 $\mathrm{g}$ for $15 \mathrm{~min}$. The supernatant was collected to measure the concentration of protein with the BCA protein assay kit (Sigma-Aldrich, 08168); the final protein concentration in each sample was adjusted to $2 \mu \mathrm{g} / \mu \mathrm{L}$. From each sample $20 \mu \mathrm{g}$ of total protein was taken for SDS-PAGE electrophoresis; $6 \times$ sodium dodecyl sulfate (SDS) protein loading buffer was added. The protein was denatured by heating at $100^{\circ} \mathrm{C}$ for $10 \mathrm{~min}$, and separated using SDS polyacrylamide gel electrophoresis (SDSPAGE). 
Protein was transferred onto a polyvinylidene fluoride membrane using a wet transfer method. The membrane was blocked in 5\% (w/v) skimmed milk for $3 \mathrm{~h}$, washed with Tris-buffered saline with Tween (TBST), and then incubated with the main target gene antibody (Hua'an, Hangzhou, China) at $4^{\circ} \mathrm{C}$ overnight. The next day, the membrane was washed with TBST and incubated with the corresponding second antibody: rabbit IgG (Cell Signaling Technology, 3900S) for $1 \mathrm{~h}$ at room temperature. The protein on the membrane was colored using ECL and the western blot system (Amersham, 32209). $\beta$-action was taken as an internal reference protein. Before the formal experiment, the marker of the target protein was analyzed to detect the target band (Thermo, 26616).

Library construction, transcriptome sequencing and analysis. Gonad tissues (from nine male or female fish) were removed from storage at $-80^{\circ} \mathrm{C}$, cut into two small pieces under liquid nitrogen, and used for transcriptome and proteome sequencing, separately. One piece was added to $1 \mathrm{~mL}$ TRIzol reagent and homogenized. The homogenate was left at room temperature for $10 \mathrm{~min}$, centrifuged at $12,000 \mathrm{~g}$ and $4^{\circ} \mathrm{C}$ for $10 \mathrm{~min}$, and the supernatant transferred to a new RNase-free $1.5 \mathrm{~mL}$ centrifuge tube. An RNeasy Micro kit (Qiagen, 74004) was used for RNA extraction and purification. Total RNA quality was tested using an Agilent 2100 instrument. Samples from three fish were combined to construct one sequencing library. Each experimental group was replicated three times. To build separate sequence libraries, male and female tilapia were analyzed separately. A total of 12 sequencing libraries were constructed: three control groups of male tilapia (M_con 1-3), three transcription knockout groups of male tilapia (M_sf1-1-3), three control groups of female tilapia (F_con 1-3), and three transcription knockout group of female tilapia ( $F_{-}$sf1 $\left.{ }^{-1} 1-3\right)$.

We followed standard Illumina Novaseq ${ }^{\mathrm{TM}} 6000$ (LC-Bio Technology CO., Ltd., Hangzhou, China) procedures for library construction and sequencing experiments. The Illumina paired-end RNA-seq method was used to sequence $M_{-}$con, $M_{-} s f 1^{-}, F_{-}$con and $F_{-}$sfl $^{-}$transcriptomes. We first obtained high-quality valid data using FastQC $^{84}$ to remove reads with adapters, reads containing $>5 \%$ base information that could not be determined, and low-quality reads (the number of bases with quality value $\mathrm{Q} \leqq 10$ accounting for more than $20 \%$ of total reads). Then, HISAT software ${ }^{85}$ was used to stitch valid data with the reference sequence for subsequent analyses. Comparison of valid data with the reference genome was performed using BLAST.

Reads per kilobase of transcript, per million mapped reads (RPKM) were used to measure the abundance of gene transcripts. DE genes were screened on the basis of their fold change and $P$ value. The $P$ value was corrected by a false discovery rate (FDR) in $R$ language ${ }^{86}$. The selection threshold 
for DE genes was fold-change $\geqq 2$ or $\leqq 0.5$, and corrected $P$-value of $<0.05$.

Library construction, proteome sequencing and analysis. We added the proper amount of SDT lysate to gonad tissue, transfer to Lysing Matrix A tube, and use Polytron homogenizer to homogenize and break (6.0 M/S, $30 \mathrm{~s}, 1-4$ times). After sonication, the sample was placed into a boiling waterbath for $10 \mathrm{~min}$, then centrifuged at 14,000 $\mathrm{g}$ for $15 \mathrm{~min}$ before the supernatant was collected and filtered with a $0.22 \mu \mathrm{m}$ centrifuge tube; the filtrate was then collected. The BCA method was used for protein quantification. Aliquot samples and store at $-20^{\circ} \mathrm{C} .20 \mu \mathrm{g}$ of protein from each experimental group was added to $6 \times$ loading buffer, then placed into a boiling waterbath for $5 \mathrm{~min} ; 12 \%$ SDS-PAGE electrophoresis (constant voltage 250 v, 40 min), Coomassie Brilliant Blue staining. Samples of each experimental group were taken $200 \mu \mathrm{g}$ protein solution, added DTT to a final concentration of 100 $\mathrm{mM}$, boiling water bath for $5 \mathrm{~min}$, and cooling to room temperature. The extraction and collection of peptides refer to the LC-Bio 's standard procedures. Samples of each experimental group were taken from $100 \mu \mathrm{g}$ peptides and labelled according to instructions on TMT labelling kits (Thermo Fisher Scientific, 90064). A total of 12 sequencing libraries were constructed: for males, three control (M_con 1-3) and three transcription knockout group (M_sf1- 1-3) groups; and for females, three control (F_con 1-3) and three treatment $\left(\mathrm{F}_{-} \mathrm{sfl}^{-1} 1-3\right)$ groups.

We mixed each group of labelled peptides and used the Agilent 1260 infinity II HPLC system for fractionation. After each sample was separated using the Easy $\mathrm{nLC}$ system with a $\mathrm{nL}$ flow rate, the Q Exactive plus mass spectrometer was used for analysis. The entire proteomics sequencing work was performed by LC-BIO (Hangzhou, China). We used Proteome Discoverer 2.2 (Thermo Fisher Scientific) software to transform and analyze original map files (raw files) generated by Q Exactive plus. Data were screened according to protein FDR $<0.01$ criteria. Proteins with fold change $>1.2$ and $P$-values (Student's t test) $<0.05$ were considered to be differentially abundant (DA) proteins.

Integrated analysis of related DE gene-DA protein pairs and functional enrichment. All related gene-protein pairs were analyzed and identified based on mRNA sequence results and Universal Protein database (https://www.uniprot.org/). Related DE gene-DA protein pairs were identified as follows: significantly DE gene with $P<0.05$, fold-change $\geqq 2$ or $\leqq 0.5$; and significantly DA protein with $P<0.05$, fold-change $\geqq 1.2$ or $\leqq 0.833$. We selected DE gene-DA protein pairs that were both up- or down-regulated for subsequent function and enrichment pathway analysis. We used heatmaps (http://www.heatmapper.ca/expression/) $)^{87}$ to intuitively analyze the distribution of related DE gene-DA protein pairs, and demonstrate quality control and differences in experimental data. 
According to the associations between DE genes and DA proteins, each small square represents a gene, and the intensity of the color represents its expression level.; the higher the expression level, the darker the color (red is up- and blue is down-regulation). Gene ontology (GO; http://www.geneontology.org) and Kyoto Encyclopedia of Genes and Genomes (KEGG) pathway (http://www.genome.jp/kegg/pathway.html) ${ }^{88}$ databases were used to assign terms and pathways to the DE mRNA-DA protein pairs to investigate their potential biological functions.

Hematoxylin-eosin (HE) staining. Gonad tissues were fixed in $4 \%$ paraformaldehyde for $4 \mathrm{~d}$, then washed several times with PBS, dehydrated with an alcohol gradient, cleared with xylene, and soaked and embedded in paraffin. To prepare sections, $5 \mu \mathrm{m}$ slices were cut using a microtome. Paraffin slices were routinely dewaxed, stained with hematoxylin for $7 \mathrm{~min}$, washed with tap water and then warm water for $1 \mathrm{~min}$, immersed in $1 \%$ hydrochloric acid alcohol for about $60 \mathrm{~s}$, and then stained with eosin solution for $5 \mathrm{~min}$. The HE-stained sections were dehydrated with an alcohol gradient, cleared with xylene, and sealed with neutral resin. Gonad sections were observed under a microscope (Leica UB203I, Nussloch, Germany) to detect pathological changes, and photographed.

Determination of serum hormone. We used an enzyme-linked immunoassay kit to determine serum gonadotropin releasing hormone (GnRH, Nanjing Jiancheng, H297), follicle stimulating hormone (FSH, Nanjing Jiancheng, H101-1-2), luteinizing hormone (LH, Nanjing Jiancheng, H206-1-2), estradiol ( $E_{2}$, Nanjing Jiancheng, H102), and testosterone (T, Nanjing Jiancheng, H090-1-2) levels in fish, females and males. All kits were purchased from Nanjing Jiancheng Institute of Biological Engineering (Nanjing, China). Hormone concentrations in serum were determined following kit instructions; each sample was repeated three times. Standard kit products were first diluted according to the gradient of 16: 8: 4: 2: 1 to prepare standards of different concentrations for drawing the standard curve. A sample of $40 \mu \mathrm{L}$ of the serum to be tested was taken and related parameters were determined according to kit operating procedures. Results were read by a Multiskan spectrum microplate spectrophotometer (BioTek Eon, USA). Different hormone concentrations in samples in each group were determined at different wavelengths, and corresponding sample concentrations were calculated according to the linear regression equation of the standard curve.

Statistics and reproducibility. The three batches of eggs in the sfl transcription knockout experiment produced 314 experimental fish in vivo. We mainly report on the first batch of experimental results. Treatment and control group fish contained at least three biological replicates. In transcriptome and proteomics sequencing, three biological replicates were included for each 
treatment. For detailed information on statistical samples, see respective figure legends. Different statistical methods are used for variance and multiple comparisons; see relevant figure legends for specific statistical techniques.

Reporting summary. A detailed experimental design and results are available in the Nature Research Reporting Summary linked to this article, and in supplementary files.

\section{Data availability}

Raw sequencing data in the mRNA library has been assigned the GEO accession number GSE161135 (https://www.ncbi.nlm.nih.gov/geo/query/acc.cgi?acc=GSE161135). Mass spectrometry proteomics data have been deposited in the ProteomeXchange Consortium (http://proteomecentral.proteomexchange.org) via the iProX partner repository ${ }^{89}$ with the dataset identifier PXD022939 (http://proteomecentral.proteomexchange.org/cgi/GetDataset?ID= PXD022939). Source data for Fig 3c-h, 4a, 4b; and Figs 2a, b; 3a, b; 4a, b are provided as Source Data files.

\section{Funding}

The study was supported financially by Central Public-interest Scientific Institution Basal Research Fund, CAFS (NO. 2020JBFM04), and National Natural Science Foundation of China (NO. 32002363). The funding body provided played a vital role in the design of this study and collection of samples.

\section{Author contributions}

X.P., C.Z.M and Q.J. conceived and designed the experiment; C.Z.M and Q.J. carried out antisense RNA sequence design and transfection; Q.J., B.J.W. and T.Y.F. extracted RNA and constructed libraries. H.J. and B.J.W. conducted the reproduction experiment. Z.H.J and L.H.X sampled gonad tissues and prepared sections for microscopy. T.Y.F. and B.J.W verified gene mRNA expression by qRT-PCR. L.H.X detected protein expression level by western blot. C.Z.M and Q.J. uploaded experimental data and wrote the paper with contributions from all other authors. All authors have read and approved the final version of the manuscript.

\section{Acknowledgements}

We thank Steve O'Shea, PhD, from Liwen Bianji, Edanz Group China (www.liwenbianji.cn/ac), for editing the English text of various drafts of this manuscript.

\section{Competing Interests}

The authors declare that they have no competing interests. 
707 Correspondence and requests for materials should be addressed to Q.J. or X.P. 708 
Fig. 1 Detection of transfected efficiency of antisense RNA and regulation of sf1 expression in Nile tilapia gonadal tissue. a Agarose gel electrophoresis chart showing the position of target fragments in gonad tissue for each experimental group. Gonad tissue in the group transfected with antisense RNA sequence shows obvious bands at about $1000 \mathrm{bp}$ (including the plasmid $900 \mathrm{bp}+$ antisense RNA fragment about $100 \mathrm{bp}$ ). A1-7 and E1-10 marked with a green box represent the transfection experiment group $(\mathrm{n}=17$ replicates $)$; $1-7$ and D1 -4 marked with a red box are the negative control group ( $n=11$ replicates), of about 900 bp (including the plasmid 900 bp); E1-4 marked with a yellow box represent the control group $(n=4$ replicates), with no obvious band at the position of $900-1000$ bp. b, $\mathbf{c}$ Mean \pm SE sfl mRNA and sfl transcript variant X1 mRNA levels in male and female gonad tissues $(\mathrm{n}=$ 10-14 replicates). Identification of sf1 mRNA and sf1 transcript variant X1 mRNA levels in antisense RNA transfection, negative control, and control groups using qRT-PCR. $\mathrm{M}=$ male, $\mathrm{F}=$ female. $\mathrm{M}-\mathrm{Con}$ and $\mathrm{F}-\mathrm{Con}=\mathrm{M}$

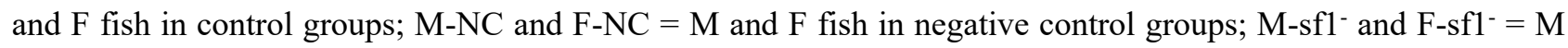
and F fish in antisense RNA transfection groups. d, e Polyacrylamide gel electrophoresis showing expression levels of sfl protein in gonad tissues of each experimental group: A1 and A2, and D1 and D2 = M and F fish in control groups; B1 and B2 and E1 and E2 = M and F fish in negative control groups; $\mathrm{C} 1-4$ and $\mathrm{F} 1-4=\mathrm{M}$ and $\mathrm{F}$ fish in antisense RNA transfection groups, respectively. Comparisons in b, $\mathbf{c}$ were analyzed by one-way ANOVA followed by Tukey's multiple comparisons test $\left({ }^{* *} P<0.01,{ }^{*} P<0.05\right)$.

\section{Fig. 2 sf1 transcription knockout inhibits development of sex organs and regulates serum hormones in Nile}

tilapia. a, b Effect of sf1 transcription knockout on external genitalia and gonad tissues of male and female Nile tilapia. According to characteristics of external genitalia, males in the control group have obviously convex urogenital; the urogenital of males with sf1 transcription knockout are strongly atrophied. Control group female fish had obvious urinary and genital openings, while the urinary and genital openings of females whose sfl transcription had been knocked out showed severe atrophy of these openings and could not be distinguished urinary or genital opening. c Representative images of HE-stained gonad tissue of male $(\times 400$, scale bar $50 \mu \mathrm{m})$ and female $(\times 100$, scale bar: $200 \mu \mathrm{m}) . \mathrm{M}=$ male, $\mathrm{F}=$ female. $\mathrm{M}-\mathrm{Con}$ and $\mathrm{F}-\mathrm{Con}=\mathrm{M}$ and $\mathrm{F}$ fish in control groups; $\mathrm{M}-\mathrm{NC}$ and $\mathrm{F}-\mathrm{NC}=$ $\mathrm{M}$ and $\mathrm{F}$ fish in negative control groups; $\mathrm{M}-\mathrm{sfl}^{-}$and $\mathrm{F}-\mathrm{sfl}^{-}=\mathrm{M}$ and $\mathrm{F}$ fish in antisense RNA transfection groups. Sp: sperm; Sg: spermatogonia; PS: primary spermatocytes; SS: second spermatocyte; St: spermatocyte. II, III, IV, and V represent oocytes of stages II, III, IV, and V, respectively. * represents vacuolation. A green box (\#\#) represents II oocyte cluster. A red box (\#) represents oocyte membrane folding. d, e Mean \pm SE serum sex hormone levels in male and female fish. Serum gonadotropin releasing hormone (GnRH), follicle stimulating hormone (FSH), luteinizing hormone $(\mathrm{LH})$, estradiol $\left(\mathrm{E}_{2}\right)$ and testosterone $(\mathrm{T})$ contents of control, negative control, and sfl 
transcription knockout groups were identified using enzyme-linked immunoassay kits. Comparisons in d, e were analyzed by one-way ANOVA followed by Tukey's multiple comparisons test $\left({ }^{*} P<0.05\right)$.

Fig. 3 sf1 transcription knockout regulates gene and protein expression profiles in gonad tissues of Nile tilapia. a, b Quantity of differentially expressed (DE) genes and differentially abundant (DA) proteins in gonad tissues of male and female tilapia in sf1 transcription knockout and control groups by transcriptome and proteome sequencing ( $n=3$ replicates). Blue represents DE genes, orange represents DA proteins; the abscissa represents quantity. $\mathbf{c}, \mathbf{e}$ DE gene-DA protein pairs in male gonad tissue between the sfl transcription knockout and control groups based on results of transcriptome sequencing ( $\mathrm{n}=3$ replicates). $\mathbf{c}$ Venn diagram showing DE genes and DA proteins, and their common DE gene-DA protein pairs with up-regulated and down-regulated transcription in males. e DE genes and DA proteins in male fish. Color codes indicate up- or down- regulated DE gene - up- or down-DA protein pairs. g Cluster analysis showing expression signatures of all DE gene-DA protein pairs in male fish. Colors represent different expressions: red shows a significant increase, blue a significant decrease. $\mathrm{M}=$ male. Pro_M- sf1- and Pro_ M-Con indicate DA proteins in sf1 transcription knockout and control groups from DE gene-DA protein pairs; Trans_M- sf1- versus Trans_M-Con indicates DE genes in both sfl transcription knockout and control groups from DE gene-DA protein pairs. d, f DE gene-DA protein pairs in gonad tissue of female fish between the sfl transcription knockout and control groups, based on results of transcriptome sequencing $(\mathrm{n}=3$ replicates $)$. $\mathbf{d}$ Venn diagram showing DE genes and DA proteins and their common DE gene-DA protein pairs with up- and downregulated transcription in females. $\mathbf{f} \mathrm{DE}$ genes and DA proteins in female fish. $\mathbf{h}$ Cluster analysis showing expression signatures of all DE gene-DA protein pairs in female fish. F = female. Pro_F-sf1- and Pro_F-Con indicate DA proteins in both sf1 transcription knockout and control groups from DE gene-DA protein pairs; Trans_ F-sf1 versus Trans_F-Con indicates DE genes in both sfl transcription knockout and control groups from DE gene-DA protein pairs. a, b False discovery rate (FDR) used to determine the threshold of $P$-values in multiple tests, calculated using corrected $P$-values. Comparisons of DA proteins in $\mathbf{a}, \mathbf{b}$ were analyzed by Student's t test.

\section{Fig. 4 sf1 transcription knockout regulates signal pathway enrichment and differential gene analysis in Nile} tilapia gonad tissue. a, b The KEGG enrichment subclass and signal pathway of DE gene-DA protein pairs in gonad tissues of male and female fish after sfl transcription knockout. Circle diameter is proportional to the quantity of DE gene-DA protein pairs in the corresponding pathway. The circle color represents the $P$ value. The $P$ value is a test of whether the proportion of DE genes between the sfl transcription knockout and control groups in the number of all genes in this KEGG pathway is greater than the proportion of DE genes in the total number of genes 
in all KEGG pathway. $P$ values in $\mathbf{a}, \mathbf{b}$ were analyzed by Student's t test. $\mathbf{c}, \mathbf{d}$ qRT-PCR verifies the expression levels of DE genes in males and females, and comparison of trends with DE gene-DA protein pairs. We selected $7 \mathrm{DE}$ genes from male and female fish for qRT-PCR verification. The trend map in $\mathbf{c}$ is calculated by $\log _{2}$ Fold change using the formula Log2Fold change (sfl transcription knockout/control). Comparisons of qRT-PCR results and sequencing results in $\mathbf{c}, \mathbf{d}$ were analyzed by square of Pearson correlation coefficient. e Schematic diagram of sfl transcription knockout inhibiting male and female Nile tilapia gonad development and regulating weight gain. hsd11b2 = hydroxysteroid (11-beta) dehydrogenase 2 ; ephx2 = soluble epoxide hydrolase 2 ; cyp4f3 = cytochrome P450 4f3; fndc1 = fibronectin type III domain-containing protein 1; capn12 = calpain 12; atp1a3b = sodium/potassium-transporting ATPase subunit alpha-3b; ugt5a1 = UDP-glucuronosyltransferase 5a1; colla2 = collagen type I alpha 2; col1a1 = collagen type I alpha 1; fn1b = fibronectin $1 \mathrm{~b}$; aldh1a2 = aldehyde dehydrogenase 1 family, member A2; ctsd = cathepsin D; ugt1ab = UDP-glucuronosyltransferase 1ab; cyp3a65= cytochrome P450 $3 a 65$.

Fig. 5 Design and action site of antisense RNA sequence of sf1. a Sequence information and interference sites of the two sfl antisense RNAs (anti-sf1-I and anti-sf1-II). The antisense anti-sf1-I sequence is 113 bp; the interference site contains the antisense sequence of a first intron partial sequence, a first exon partial sequence, and a 5'- nontranslated sequence of four bases of the sfl mRNA precursor. The antisense anti-sfl-II sequence is $104 \mathrm{bp}$; the interference site contains the antisense sequence of a second intron partial sequence, and a first exon partial sequence of the sfl mRNA precursor. b Sequence information and interference sites of the two antisense RNAs (anti-sf1 transcript variant X1-I and anti-sf1 transcript variant X1-II) of sf1 transcript variant X1. The antisense RNA sequence of the anti-sf1 transcript variant X1-I is $102 \mathrm{bp}$ in size; the interference site contains the antisense sequence of a first intron partial sequence, and a 5'-non-translated sequence of 80 bases of sfl transcript variant X1 mRNA precursor. The antisense RNA sequence of the anti-sf1 transcript variant X1-II is $102 \mathrm{bp}$ in size, and the interference site contains the antisense sequence of a second intron partial sequence, a first exon sequence, and a 5'-nontranslated sequence of 60 bases of sfl transcript variant X1 mRNA precursor. 


\section{References}

1. Hruscha A, et al. Efficient CRISPR/Cas9 genome editing with low off-target effects in zebrafish. Development. 140, 4982$4987(2013)$

2. Hwang WY, et al. Efficient genome editing in zebrafish using a CRISPR-Cas system. Nat. Biotechnol. 31, 227-229 (2013).

3. Yeh YC, et al. Using CRISPR/Cas9-mediated gene editing to further explore growth and trade-off effects in myostatinmutated F4 medaka (Oryzias latipes). Sci Rep. 7, 11435 (2017).

4. Yano. A, Nicol. B, Jouanno. E, Quillet. E, Fostier. A. The sexually dimorphic on the Y-chromosome gene (sdY) is a conserved male-specific Y-chromosome sequence in many salmonids. Evol. Appl. 6, 486-496 (2013).

5. Edvardsen RB, Sven L, Lene K, Ove SK, Anna W, Diego F. Targeted Mutagenesis in Atlantic Salmon (Salmo salar L.) Using the CRISPR/Cas9 System Induces Complete Knockout Individuals in the F0 Generation. PLoS One. 9, 108622 (2014).

6. Datsomor, A.K., Olsen, R.E., Zic, N. et al. CRISPR/Cas9-mediated editing of $\Delta 5$ and $\Delta 6$ desaturases impairs $\Delta 8$ desaturation and docosahexaenoic acid synthesis in Atlantic salmon (Salmo salar L.). Sci Rep 9, 16888 (2019).

7. Ran FA, et al. Double Nicking by RNA-Guided CRISPR Cas9 for Enhanced Genome Editing Specificity. Cell. 154, 1380$1389(2013)$

8. Parker KL, Schimmer BP. Genes essential for early events in gonadal development. Ann. Med. 34, 171-178 (2002).

9. Parker KL, Schimmer BP. The roles of the nuclear receptor steroidogenic factor 1 in endocrine differentiation and development. Trends Endocrinol. Metab. 7, 203-207 (1996).

10. Watanabe M, Tanaka M, Kobayashi D, Yoshiura Y, Oba Y, Nagahama Y. Medaka (Oryzias latipes) FTZ-F1 potentially regulates the transcription of P-450 aromatase in ovarian follicles: cDNA cloning and functional characterization. Mol. Cell. Endocrinol. 149, 221-228 (1999).

11. Lin WW, Wang HW, Sum C, Liu D, Hew CL, Chung BC. Zebrafish ftz-f1 gene has two promoters, is alternatively spliced, and is expressed in digestive organs. Biochem. J. 348, 439-446 (2000).

12. von Hofsten J, Jones I, Karlsson J, Olsson PE. Developmental expression patterns of FTZ-F1 homologues in zebrafish (Danio rerio). Gen. Comp. Endocrinol. 121, 146-155 (2001).

13. Yoshiura Y, Senthilkumaran B, Watanabe M, Oba Y, Kobayashi T, Nagahama Y. Synergistic expression of Ad4BP/SF-1 and cytochrome P-450 aromatase (ovarian type) in the ovary of Nile tilapia, Oreochromis niloticus, during vitellogenesis suggests transcriptional interaction. Biol. Reprod. 68, 1545-1553 (2003).

14. Kobayashi Y, Sunobe T, Kobayashi T, Nakamura M, Suzuki N, Nagahama Y. Molecular cloning and expression of Ad4BP/SF-1 in the serial sex changing gobiid fish, Trimma okinawae. Biochem. Biophys. Res. Commun. 332, 1073-1080 (2005) 
15. Kazeto Y, Goto-Kazeto R, Thomas P, Trant JM. Molecular characterization of three forms of putative membrane-bound progestin receptors and their tissue-distribution in channel catfish, Ictalurus punctatus. J. Mol. Endocrinol. 34, 781-791 $(2005)$

16. Choi CY, An KW, Jo PG, Kang DY, Chang YJ. Effects of gonadotropin-releasing hormone analog (GnRHa) on steroidogenic factor-1 (SF-1) and estrogen receptor beta (ERbeta) gene expression in the black porgy (Acanthopagrus schlegeli). Comp Biochem Physiol B Biochem Mol Biol. 147, 82-86 (2007).

17. Sridevi P, Dutta-Gupta A, Senthilkumaran B. Molecular Cloning and Expression Analysis of fushi tarazu Factor 1 in the Brain of Air-Breathing Catfish, Clarias gariepinus. PLoS One. 6, e28867 (2011).

18 von Hofsten J, Karlsson J, Jones I, Olsson PE. Expression and regulation of fushi tarazu factor-1 and steroidogenic genes during reproduction in arctic char (Salvelinus alpinus). Biol. Reprod. 67, 1297-1304 (2002).

19. von Hofsten. J, Larsson. A, Olsson. PE. Novel steroidogenic factor-1 homolog (ffld) is coexpressed with anti-Mullerian hormone $(A M H)$ in zebrafish. Dev. Dyn. 233, 595-604 (2010).

20. Wang D-S, et al. Foxl2 up-regulates aromatase gene transcription in a female-specific manner by binding to the promoter as well as interacting with Ad4 binding protein/steroidogenic factor 1. Mol. Endocrinol. 21, 712-725 (2007).

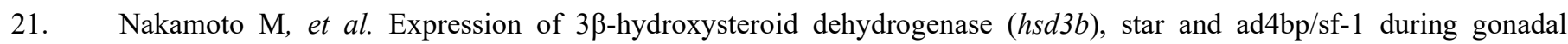
development in medaka (Oryzias latipes). Gen Comp Endocrinol. 176, 222-230 (2012).

22. Xie Q-P, He X, Sui Y-N, Chen L-L, Sun L-N, Wang D-S. Haploinsufficiency of SF-1 Causes Female to Male Sex Reversal in Nile Tilapia, Oreochromis niloticus. Endocrinology. 157, 2500-2514 (2016).

23. Tomizawa J, Itoh T, Selzer G, Som T. Inhibition of ColE1 RNA primer formation by a plasmid-specified small RNA. Proc. Natl. Acad. Sci. U. S. A. 78, 1421-1425 (1981).

24. Oeller PW, Wong LM, Taylor LP, Pike DA, Theologis A. Reversible inhibition of tomato fruit senescence by antisense RNA. Science. 254, 437-439 (1991).

25. Cuozzo, M., O'Connell, K., Kaniewski, W. et al. Viral Protection in Transgenic Tobacco Plants Expressing the Cucumber Mosaic Virus Coat Protein or its Antisense RNA. Nat Biotechnol 6, 549-557 (1988).

26. Örvar BL, Ellis BE. Transgenic tobacco plants expressing antisense RNA for cytosolic ascorbate peroxidase show increased susceptibility to ozone injury. Plant J. 11, 1297-1305(1997).

27. Fish JE, et al. Hypoxia-inducible expression of a natural cis-antisense transcript inhibits endothelial nitric-oxide synthase. J. Biol. Chem. 282, 15652-15666 (2007).

28. Harshe RP, et al. Endogenous antisense RNA curbs CD39 expression in Crohn's disease. Nat. Commun. 11, 5894-5894 (2020).

29. El-Khairi R, Achermann JC. Steroidogenic Factor-1 and Human Disease. Semin. Reprod. Med. 30, 374-381 (2012). 
30. Majdic G, et al. Knockout mice lacking steroidogenic factor 1 are a novel genetic model of hypothalamic obesity. Endocrinology. 143, 607-614 (2002).

31 Zhao LP, Bakke M, Parker KL. Pituitary-specific knockout of steroidogenic factor 1. Mol. Cell. Endocrinol. 185, 2732(2001).

32. Schwetz V, Gumpold R, Graupp M, Hacker N, Obermayer-Pietsch B. Osteocalcin is not a strong determinant of serum testosterone and sperm count in men from infertile couples. Andrology. 1, 590-594 (2013).

33. Caron KM, Clark BJ, Ikeda Y, Parker KL. Steroidogenic factor 1 acts at all levels of the reproductive axis. Steroids. 62, 53-56 (1997).

34. Tang B, Hu W, Hao J, Zhu Z. Developmental expression of steroidogenic factor-1, cyp19ala and cyp19a1b from common carp (Cyprinus carpio). Gen. Comp. Endocrinol. 167, 408-416 (2010).

35. Leers-Sucheta S, Morohashi KI, Mason JI, Melner MH. Synergistic activation of the human type II 3beta-hydroxysteroid dehydrogenase/delta5-delta4 isomerase promoter by the transcription factor steroidogenic factor-1/adrenal 4-binding protein and phorbol ester. J. Biol. Chem. 272, 7960-7967 (1997).

36. Brandt T, et al. 46,XY disorder of sex development and developmental delay associated with a novel 9q33.3 microdeletion encompassing NR5A1. Eur. J. Med. Genet. 56, 619-623 (2013).

37. Guchhait R, Chatterjee A, Mukherjee D, Pramanick K. Seasonal ovarian development in relation to the gonadotropins, steroids, aromatase and steroidogenic factor $1(S F-1)$ in the banded gourami, Trichogaster fasciata. Gen. Comp. Endocrinol. 268, 40-49 (2018).

38. Dewailly D, Robin G, Peigne M, Decanter C, Pigny P, Catteau-Jonard S. Interactions between androgens, FSH, antiMullerian hormone and estradiol during folliculogenesis in the human normal and polycystic ovary. Hum. Reprod. Update. 22, 709-724 (2016).

39. Nagler JJ, Idler DR. Invitro ovarian estradiol-17-beta and testosterone responses to pituitary extract and corresponding serum levels during the prespawning to vitellogenic phases of the reproductive-cycle in winter flounder (pseudopleuronectes-americanus). Comp. Biochem. Physiol. A. 101, 69-75 (1992).

40. Duval DL, Nelson SE, Clay CM. A binding site for steroidogenic factor-1 is part of a complex enhancer that mediates expression of the murine gonadotropin-releasing hormone receptor gene. Biol. Reprod. 56, 160-168 (1997).

41. Halvorson LM, Kaiser UB, Chin WW. Stimulation of luteinizing hormone beta gene promoter activity by the orphan nuclear receptor, steroidogenic factor-1. J. Biol. Chem. 271, 6645-6650 (1996).

42. Ingraham HA, et al. The nuclear receptor steroidogenic factor-1 acts at multiple levels of the reproductive axis. Genes Dev. 8, 2302-2312 (1994).

43. Brown P, McNeilly AS. Steroidogenic factor-1 ( $S F-1)$ and the regulation of expression of luteinising hormone and follicle 
stimulating hormone b-subunits in the sheep anterior pituitary in vivo. Int. J. Biochem. Cell Biol. 29, 1513-1524 (1997).

44. Devlin RH, Nagahama Y. Sex determination and sex differentiation in fish: an overview of genetic, physiological, and environmental influences. Aquaculture. 208, 191-364 (2002).

45. Choi CY, An KW, Jo PG, Kang DY, Chang YJ. Effects of gonadotropin-releasing hormone analog (GnRHa) on steroidogenic factor-1 $(S F-1)$ and estrogen receptor beta (ER beta) gene expression in the black porgy (Acanthopagrus schlegeli). Comp. Biochem. Physiol. B-Biochem. Mol. Biol. 147, 82-86 (2007).

RanjithRamasamy MD, et al. High serum FSH levels in men with nonobstructive azoospermia does not affect success of microdissection testicular sperm extraction. Fertil. Steril. 92, 590-593(2009)

47. Livera G, Rouiller-Fabre V, Pairault C, Levacher C, Habert R. Regulation and perturbation of testicular functions by vitamin A. Reproduction. 124, 173-180 (2002).

48. Li H, Palczewski K, Baehr W, Clagett-Dame M. Vitamin A Deficiency Results in Meiotic Failure and Accumulation of Undifferentiated Spermatogonia in Prepubertal Mouse Testis. Biol. Reprod. 84, 336-341 (2011).

49. Brown JA, Eberhardt DM, Schrick FN, Roberts MP, Godkin JD. Expression of retinol-binding protein and cellular retinolbinding protein in the bovine ovary. Mol. Reprod. Dev. 64, 261-269 (2003).

50. Buckley DB, Klaassen CD. Induction of Mouse UDP-Glucuronosyltransferase mRNA Expression in Liver and Intestine by Activators of Aryl-Hydrocarbon Receptor, Constitutive Androstane Receptor, Pregnane X Receptor, Peroxisome Proliferator-Activated Receptor alpha, and Nuclear Factor Erythroid 2-Related Factor 2. Drug Metab. Dispos. 37, 847856 (2009).

51. Huang H, Wu Q. Cloning and Comparative Analyses of the Zebrafish Ugt Repertoire Reveal Its Evolutionary Diversity. PLoS One. 5, e9144 (2010).

52. Christen V, Fent K. Tissue-, sex- and development-specific transcription profiles of eight UDP-glucuronosyltransferase genes in zebrafish (Danio rerio) and their regulation by activator of aryl hydrocarbon receptor. Aquat. Toxicol. 150, 93$102(2014)$

53. Yasuhiko K, Angel R, R Marina R, Concepción R-E, Juan Carlos IB. Retinoic acid signalling links left-right asymmetric patterning and bilaterally symmetric somitogenesis in the zebrafish embryo. Nature. 435, 165-171 (2005).

54. Smets KJ, Barlow T, Vanhaesebrouck P. Maternal vitamin A deficiency and neonatal microphthalmia: complications of biliopancreatic diversion? Eur. J. Pediatr. 165, 502-504 (2006).

55. Sadoul B, Geffroy B. Measuring cortisol, the major stress hormone in fishes. J. Fish Biol. 94, 540-555 (2019).

56. Li M, et al. Retinoic acid triggers meiosis initiation via stra8-dependent pathway in Southern catfish, Silurus meridionalis. Gen. Comp. Endocrinol. 232, 191-198 (2016).

57. Feng R, et al. Retinoic acid homeostasis through aldh1a2 and cyp26a1 mediates meiotic entry in Nile tilapia (Oreochromis 
niloticus). Sci Rep. 5, 10131 (2015).

58. Mommsen TP, Vijayan MM, Moon TW. Cortisol in teleosts: dynamics, mechanisms of action, and metabolic regulation. Rev. Fish. Biol. Fish. 9, 211-268 (1999).

59. Yamaguchi T, Yoshinaga N, Yazawa T, Gen K, Kitano T. Cortisol Is Involved in Temperature-Dependent Sex Determination in the Japanese Flounder. Endocrinology. 151, 3900-3908 (2010).

60. Zygmunt K. The 11ß-hydroxysteroid dehydrogenases: functions and physiological effects. Mol. Cell. Endocrinol. 151, 121-127 (1999).

61. Alderman. SL, Vijayan. MM. 11 -Hydroxysteroid dehydrogenase type 2 in zebrafish brain: a functional role in hypothalamus-pituitary-interrenal axis regulation. J. Endocrinol. 215, 393 (2012).

62. Tokarz J, Norton W, Moeller G, de Angelis MH, Adamski J. Zebrafish 20 beta-Hydroxysteroid Dehydrogenase Type 2 Is Important for Glucocorticoid Catabolism in Stress Response. PLoS One. 8, e54851 (2013).

63. Hu YC, Chu K-F, Hwang L-Y, Lee T-H. Cortisol regulation of Na+, K+-ATPase beta 1 subunit transcription via the prereceptor 11 beta-hydroxysteroid dehydrogenase 1-like (11 beta-Hsd1L) in gills of hypothermal freshwater milkfish, $J$. Steroid Biochem. Mol. Biol. 192, 105381 (2019).

64 Fernandino JI, Hattori RS, Kishii A, Strüssmann CA, Somoza GM. The cortisol and androgen pathways cross talk in high temperature-induced masculinization: The 11ß-hydroxysteroid dehydrogenase as a key enzyme. Endocrinology, 153, 6003-6011(2012)

65. Presley CJ, et al. A new approach to understanding racial disparities in prostate cancer treatment. J. Geriatr. Oncol. 4, 1$8(2013)$

66. Das DK, et al. miR-1207-3p regulates the androgen receptor in prostate cancer via FNDC1/fibronectin. Exp. Cell Res. 348, 190-200 (2016).

67. Jansen J, Greither T, Behre HM. Androgen-Regulated microRNAs (AndroMiRs) as Novel Players in Adipogenesis. I $n t$. J. Mol. Sci. 20, 5767 (2019).

68. Kopelman PG. Obesity as a medical problem. Nature. 404, 635-643 (2000).

69. Bland ML, et al. Haploinsufficiency of steroidogenic factor-1 in mice disrupts adrenal development leading to an impaired stress response. Proc. Natl. Acad. Sci. U. S. A. 97, 14488-14493 (2000).

70. Blanco G, Mercer RW. Isozymes of the Na-K-ATPase: heterogeneity in structure, diversity in function. Am. J. Physiol. 275, 633-650 (1998).

71. Ollinger K. Inhibition of cathepsin D prevents free-radical-induced apoptosis in rat cardiomyocytes. Arch. Biochem. Biophys. 373, 346-351 (2000).

72. Johansson AC, Steen H, Ollinger K, Roberg K. Cathepsin D mediates cytochrome c release and caspase activation in 
human fibroblast apoptosis induced by staurosporine. Cell Death Differ. 10, 1253-1259 (2003).

73. Siklos M, BenAissa M, Thatcher GRJ. Cysteine proteases as therapeutic targets: does selectivity matter? A systematic review of calpain and cathepsin inhibitors. Acta Pharm Sin B. 5, 506-519 (2015).

74. Bukowska A, Lendeckel U, Bode-Boeger SM, Goette A. Physiologic and Pathophysiologic Role of Calpain: Implications for the Occurrence of Atrial Fibrillation. Cardiovasc. Ther. 30, e115-e127 (2012).

75. Grigorakis K, Alexis MN. Effects of fasting on the meat quality and fat deposition of commercial-size farmed gilthead sea bream (Sparus aurata, L.) fed different dietary regimes. Aquac. Nutr. 11, 341-344 (2005).

76. Johnson RB. Lipid Deposition in Oocytes of Teleost Fish During Secondary Oocyte Growth. Rev Fisher Sci. 17, 78-89 (2009).

77. Hu J, Zhang Z, Shen W-J, Azhar S. Cellular cholesterol delivery, intracellular processing and utilization for biosynthesis of steroid hormones. Nutr. Metab. 7, 1-25 (2010).

78. Hengstler JG, Stewart JD, Bolt HM. Epoxide hydrolases are not only a molecular sponge sucking up genotoxic epoxides: new roles in control of blood pressure, inflammation as well as nociception and cell proliferation. Arch. Toxicol. 83, 289291 (2009).

79. Norwood S, Liao J, Hammock BD, Yang GY. Epoxyeicosatrienoic acids and soluble epoxide hydrolase: potential therapeutic targets for inflammation and its induced carcinogenesis. Am. J. Transl. Res. 2, 447-457 (2010).

80. Shkolnik K, Ben-Dor S, Galiani D, Hourvitz A, Dekel N. A novel ovary-specific and ovulation-associated variant of epoxide hydrolase 2. FEBS Lett. 581, 4891-4898 (2007).

81. Decker M, Arand M, Cronin A. Mammalian epoxide hydrolases in xenobiotic metabolism and signalling. Arch. Toxicol. 83, 297-318 (2009).

82. Trinh LA, Stainier DYR. Fibronectin regulates epithelial organization during myocardial migration in zebrafish. Dev. Cell. 6, 371-382 (2004).

83. Chiu CH, Chou CW, Takada S, Liu YW. Development and Fibronectin Signaling Requirements of the Zebrafish Interrenal Vessel. PLoS One. 7, e43040 (2012).

84. Andrews S. FastQC: a quality control tool for high throughput sequence data. Babraham Bioinformatics (2010).

85. Kim D, Langmead B, Salzberg, SL. HISAT: a fast spliced aligner with low memory requirements. Nat. Methods 12, 357$360(2015)$.

86. Frazee AC. et al. Ballgown bridges the gap between transcriptome assembly and expression analysis. Nat. Biotechnol. 33, $243-246(2015)$

87. Babicki S. et al. 2016. Heatmapper: web-enabled heat mapping for all. Nucleic Acids Res. 44, 147-153(2016).

88 Kanehisa M. et al. KEGG for linking genomes to life and the environment. Nucleic Acids Res. 36, 480-484 (2008). 
89. Ma J, et al. iProX: an integrated proteome resource. Nucleic Acids Res. 47, D1211-D1217(2019). 


\section{Figures}
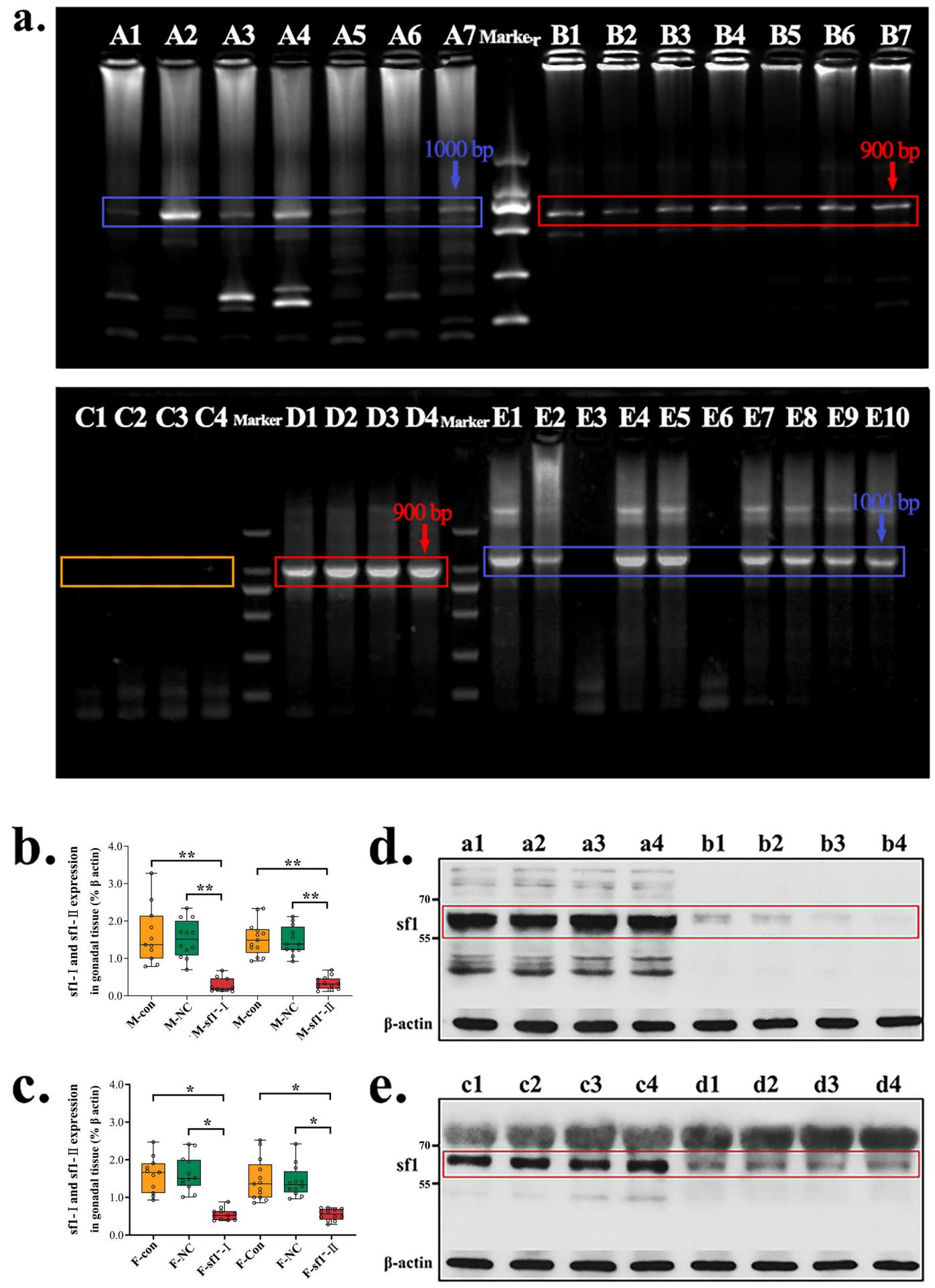

Figure 1

Detection of transfected efficiency of antisense RNA and regulation of sf 1 expression in Nile tilapia gonadal tissue. a Agarose gel electrophoresis chart showing the position of target fragments in gonad tissue for each experimental group. Gonad tissue in the group transfected with antisense RNA sequence 
shows obvious bands at about $1000 \mathrm{bp}$ (including the plasmid $900 \mathrm{bp}+$ antisense RNA fragment about $100 \mathrm{bp}$ ). A1-7 and E1-10 marked with a green box represent the transfection experiment group $(\mathrm{n}=17$ replicates); B1-7 and D1-4 marked with a red box are the negative control group ( $n=11$ replicates), of about $900 \mathrm{bp}$ (including the plasmid $900 \mathrm{bp}$ ); E1-4 marked with a yellow box represent the control group ( $n=4$ replicates), with no obvious band at the position of 900-1000 bp. b, c Mean \pm SE sf1 mRNA and sf1 transcript variant X1 mRNA levels in male and female gonad tissues ( $n=10-14$ replicates). Identification of sf1 mRNA and sf1 transcript variant X1 mRNA levels in antisense RNA transfection, negative control, and control groups using qRT-PCR. $\mathrm{M}=$ male, $\mathrm{F}=$ female. $\mathrm{M}-\mathrm{Con}$ and $\mathrm{F}-\mathrm{Con}=\mathrm{M}$ and $\mathrm{F}$ fish in control groups; M-NC and F-NC = M and F fish in negative control groups; M-sf1- and F-sf1- = M and $\mathrm{F}$ fish in antisense RNA transfection groups. d, e Polyacrylamide gel electrophoresis showing expression levels of sf1 protein in gonad tissues of each experimental group: A1 and A2, and D1 and D2 $=\mathrm{M}$ and $\mathrm{F}$ fish in control groups; $\mathrm{B} 1$ and $\mathrm{B} 2$ and $\mathrm{E} 1$ and $\mathrm{E} 2$ = $\mathrm{M}$ and $\mathrm{F}$ fish in negative control groups; $\mathrm{C} 1-$ 4 and $\mathrm{F} 1-4=\mathrm{M}$ and $\mathrm{F}$ fish in antisense RNA transfection groups, respectively. Comparisons in $\mathrm{b}, \mathrm{c}$ were analyzed by one-way ANOVA followed by Tukey's multiple comparisons test $(P<0.01, P<0.05)$.
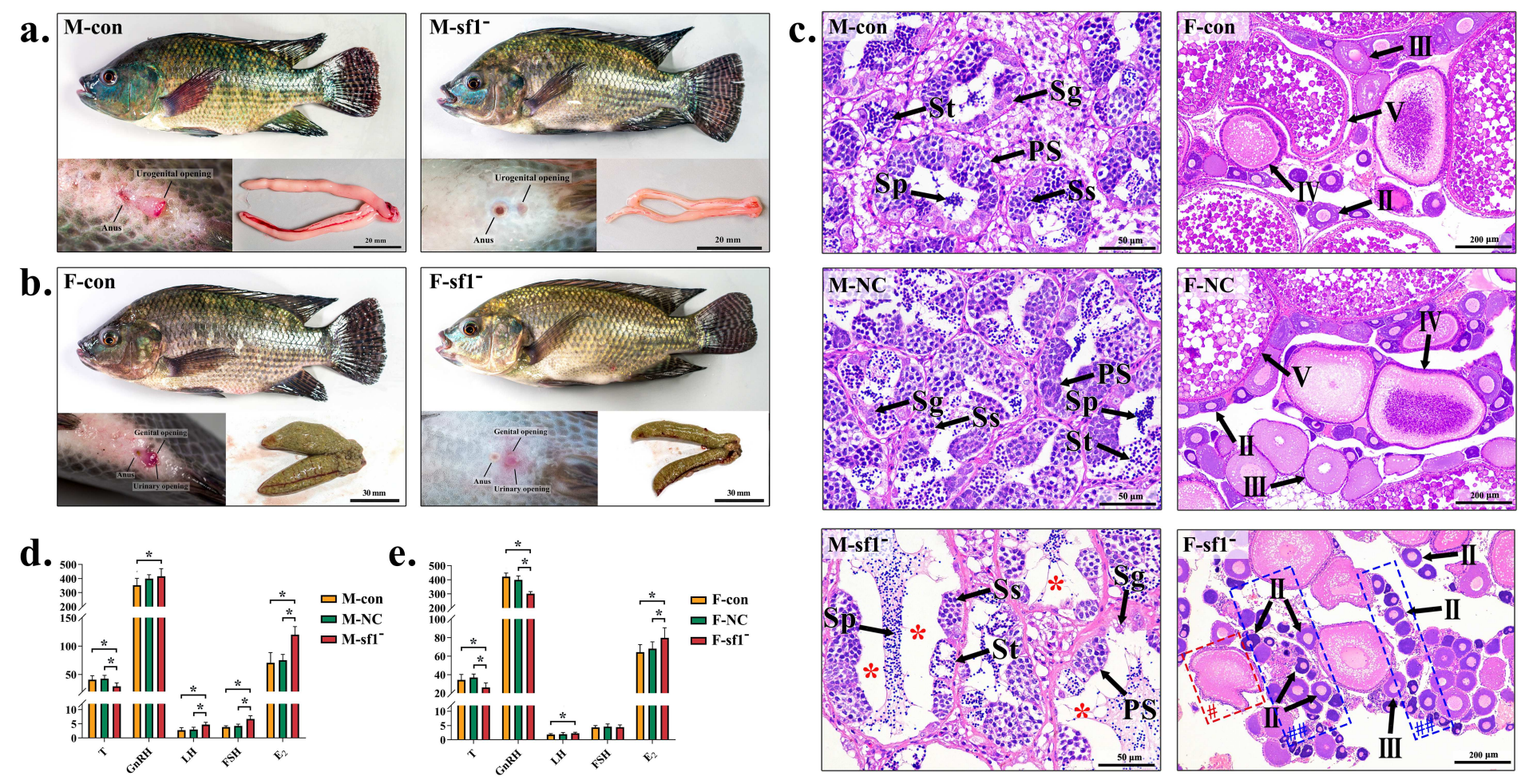

\section{Figure 2}

sf1 transcription knockout inhibits development of sex organs and regulates serum hormones in Nile tilapia. a, b Effect of sf1 transcription knockout on external genitalia and gonad tissues of male and female Nile tilapia. According to characteristics of external genitalia, males in the control group have obviously convex urogenital; the urogenital of males with sf1 transcription knockout are strongly atrophied. Control group female fish had obvious urinary and genital openings, while the urinary and genital openings of females whose sf1 transcription had been knocked out showed severe atrophy of 
these openings and could not be distinguished urinary or genital opening. c Representative images of HEstained gonad tissue of male $(\times 400$, scale bar $50 \mu \mathrm{m})$ and female $(\times 100$, scale bar: $200 \mu \mathrm{m})$. $\mathrm{M}=$ male, $\mathrm{F}$ $=$ female. $\mathrm{M}$-Con and F-Con $=\mathrm{M}$ and $\mathrm{F}$ fish in control groups; $\mathrm{M}-\mathrm{NC}$ and $\mathrm{F}-\mathrm{NC}=\mathrm{M}$ and $\mathrm{F}$ fish in negative control groups; M-sf1- and F-sf1- = M and F fish in antisense RNA transfection groups. Sp: sperm; Sg:

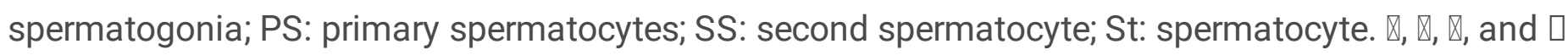

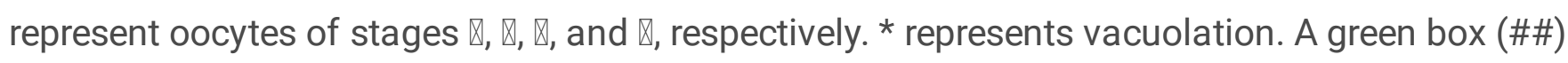
represents $₫$ oocyte cluster. A red box (\#) represents oocyte membrane folding. $d$, e Mean \pm SE serum sex hormone levels in male and female fish. Serum gonadotropin releasing hormone $(\mathrm{GnRH})$, follicle stimulating hormone (FSH), luteinizing hormone (LH), estradiol (E2) and testosterone (T) contents of control, negative control, and sf1 transcription knockout groups were identified using enzyme-linked immunoassay kits. Comparisons in d, e were analyzed by one-way ANOVA followed by Tukey's multiple comparisons test $\left({ }^{*} \mathrm{P}<0.05\right)$. 


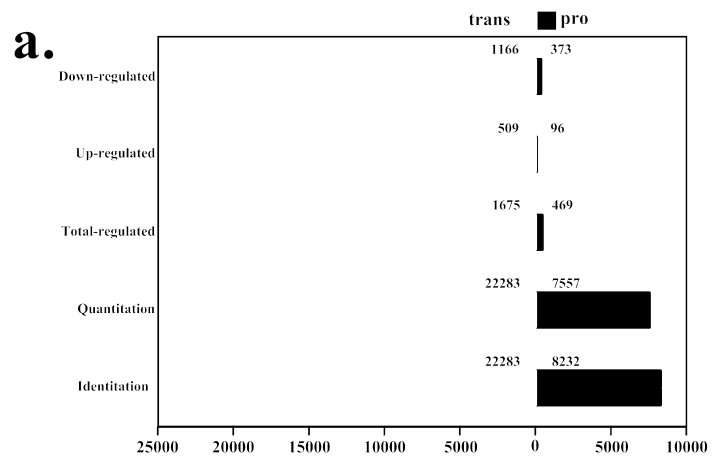

c.

b.

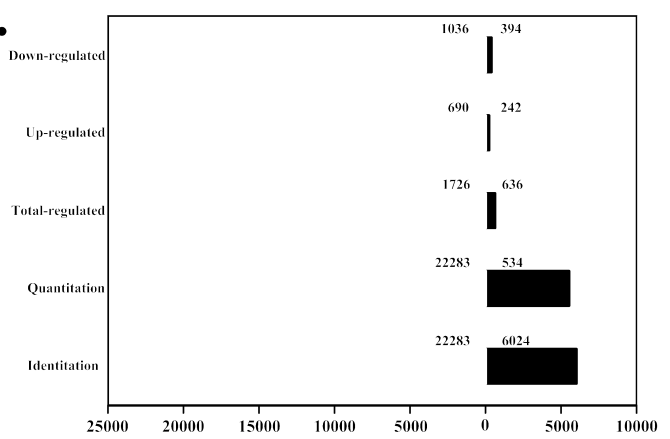

d.

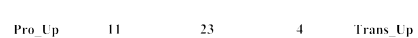

Pro_t e.

Trans Lip

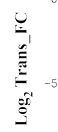

f.

Log $_{2}$ Pro $F$ F

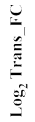

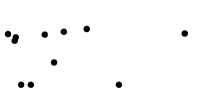

Diff_state

Com_Dom

- Trans_domproulu

Trans_Up_Pro_Dow

$\log _{2}$ Pro_fCC g.

g.

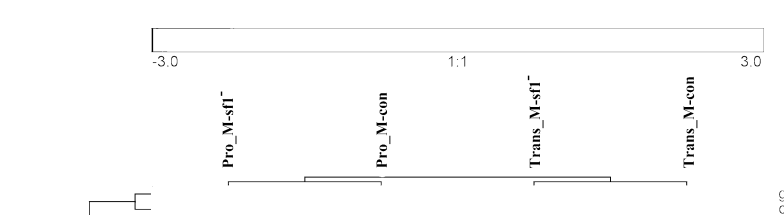

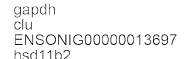

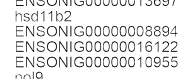
ENSONIG0000001095
nol9
cyp4f3 ( 1 of many) Cyp4f3 ( 1 of many)
ENSONIG0000000792
AKR1D1 ( 1 of many) ephx2 ENSONIGO0000001810 lck
akrib1
COMTD1 1 of many) gyg1a 1 of many) cypax
brox nde1 1
ENSONIG00000004507

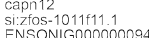
ENSONIGO00000009460
ENSONIG00000008413 mXra5b 0 ENONIGO0000001697 psmb8a cpa1
stoml3b f8
atp 1 a $3 b$
nadll (im: 13.2535 (1 of many)
inSONIG0000001913) ENSONIG000000005382 gnai2a TOM1 1 ( 1 of many) Syn2b ENSONIG0000001656 syn1 1 (1 of many)
mrcib ( ugt5a1
abcb11a pdzd3b eet2a. 2 h.

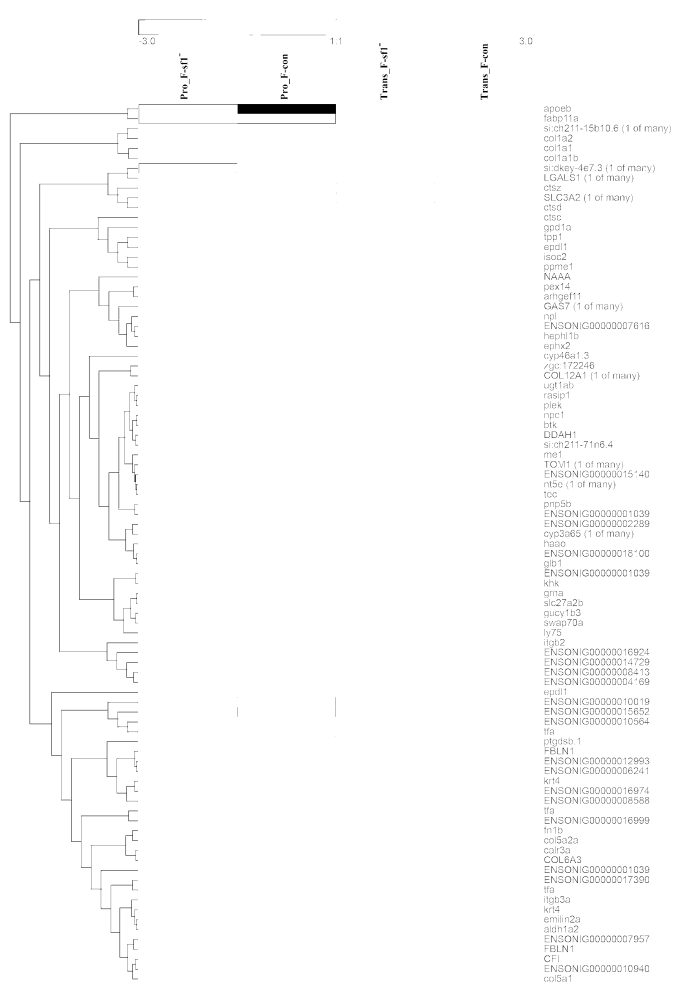

\section{Figure 3}

sf1 transcription knockout regulates gene and protein expression profiles in gonad tissues of Nile tilapia. $a, b$ Quantity of differentially expressed (DE) genes and differentially abundant (DA) proteins in gonad tissues of male and female tilapia in sf1 transcription knockout and control groups by transcriptome and proteome sequencing ( $n=3$ replicates). Blue represents DE genes, orange represents DA proteins; the abscissa represents quantity. c, e DE gene-DA protein pairs in male gonad tissue between the sf1 
transcription knockout and control groups based on results of transcriptome sequencing $(n=3$ replicates). c Venn diagram showing DE genes and DA proteins, and their common DE gene-DA protein pairs with up-regulated and down-regulated transcription in males. e DE genesand DA proteins in male fish. Color codes indicate up- or down- regulated DE gene - up- or down-DA protein pairs. g Cluster analysis showing expression signatures of all DE gene-DA protein pairs in male fish. Colors represent different expressions: red shows a significant increase, blue a significant decrease. $M=$ male. Pro_M- sf1and Pro_ M-Con indicate DA proteins in sf1 transcription knockout and control groups from DE gene-DA protein pairs; Trans_ M- sf1-versus Trans_ M-Con indicates DE genes in both sf1 transcription knockout and control groups from DE gene-DA protein pairs. $d, f D E$ gene-DA protein pairs in gonad tissue of female fish between the sf1 transcription knockout and control groups, based on results of transcriptome sequencing ( $n=3$ replicates). $d$ Venn diagram showing DE genes and DA proteins and their common DE gene-DA protein pairs with up- and down- regulated transcription in females. $f$ DE genes and DA proteins in female fish. $h$ Cluster analysis showing expression signatures of all DE gene-DA protein pairs in female fish. F = female. Pro_F-sf1- and Pro_F-Con indicate DA proteins in both sf1 transcription knockout and control groups from DE gene-DA protein pairs; Trans_ F-sf1-versus Trans_ F-Con indicates DE genes in both sf1 transcription knockout and control groups from DE gene-DA protein pairs. a, b False discovery rate (FDR) used to determine the threshold of P-values in multiple tests, calculated using corrected P-values. Comparisons of DA proteins in a, b were analyzed by Student's t test. 
a.
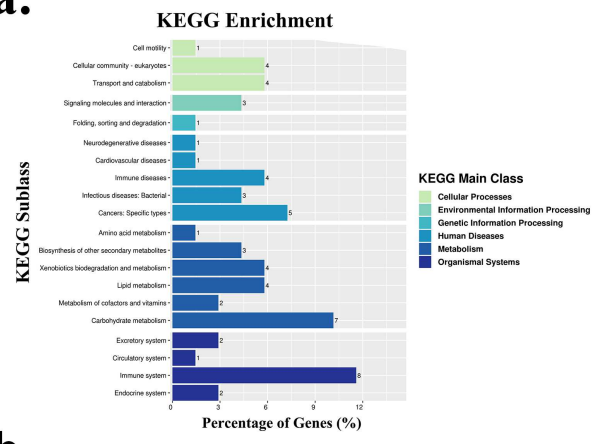

b.
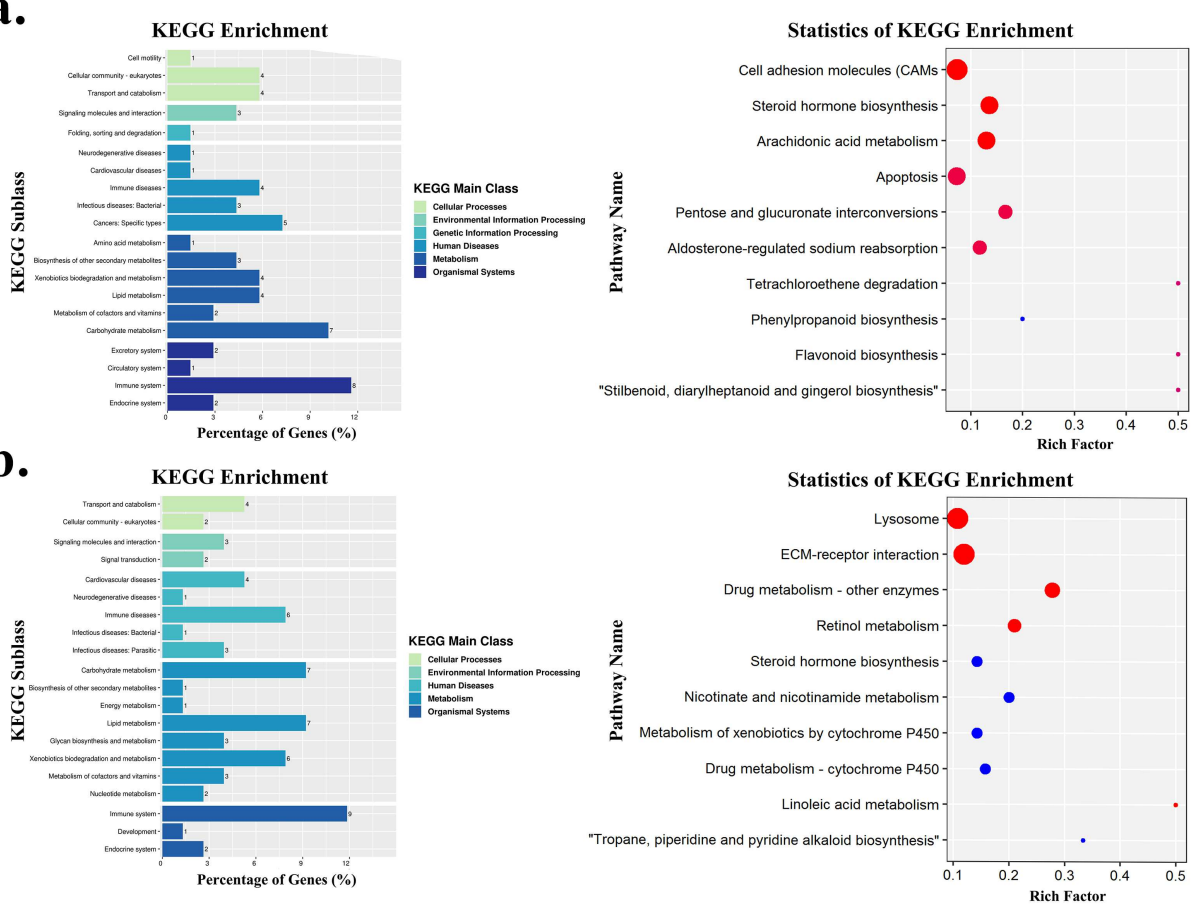

e.

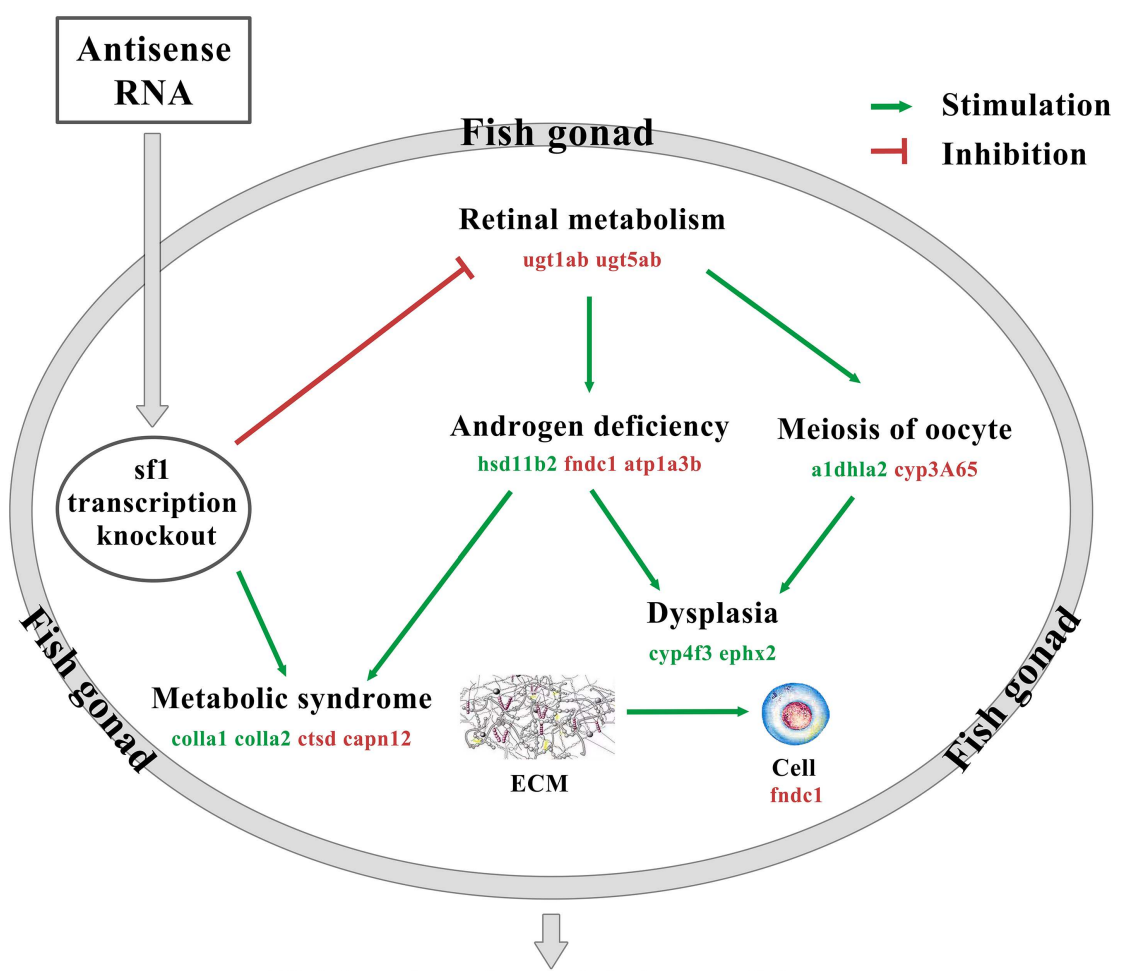

Weight gain and gonadal abnormalities

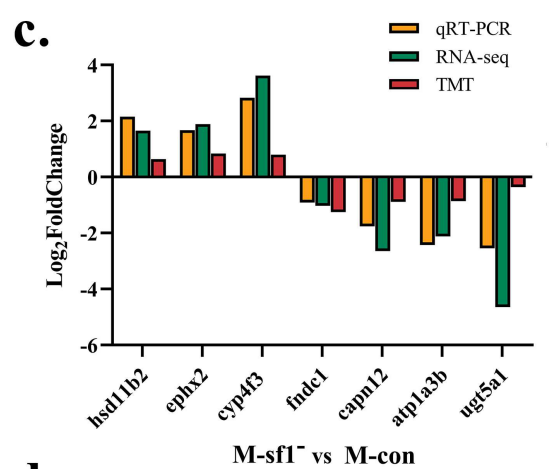

d.

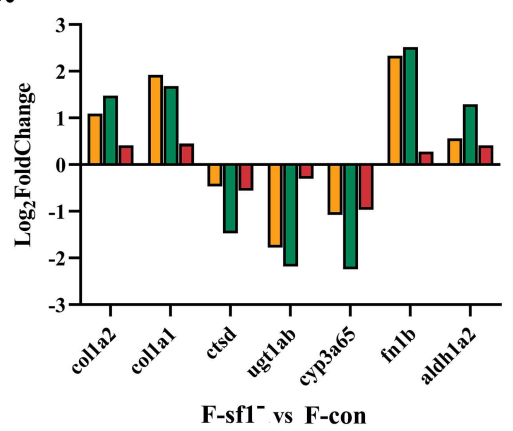


transcription knockout and control groups in the number of all genes in this KEGG pathway is greater than the proportion of DE genes in the total number of genes in all KEGG pathway. $P$ values in $a, b$ were analyzed by Student's t test. c, d qRT-PCR verifies the expression levels of DE genes in males and females, and comparison of trends with DE gene-DA protein pairs. We selected $7 \mathrm{DE}$ genes from male and female fish for qRT-PCR verification. The trend map in $\mathrm{c}$ is calculated by Log2Fold change using the formula Log2Fold change (sf1 transcription knockout/control). Comparisons of qRT-PCR results and sequencing results in $c, d$ were analyzed by square of Pearson correlation coefficient. e Schematic diagram of sf1 transcription knockout inhibiting male and female Nile tilapia gonad development and regulating weight gain. hsd11b2 = hydroxysteroid (11-beta) dehydrogenase 2 ; ephx2 = soluble epoxide hydrolase 2; cyp4f3 = cytochrome P450 4f3; fndc1 = fibronectin type III domain-containing protein 1; capn12 = calpain 12; atp $1 \mathrm{a} 3 \mathrm{~b}=$ sodium/potassium-transporting ATPase subunit alpha-3b; ugt5a1 = UDPglucuronosyltransferase $5 \mathrm{a} 1$; col1 $\mathrm{a} 2$ = collagen type I alpha 2 ; col1 $1 \mathrm{a} 1=$ collagen type $\mathrm{I}$ alpha $1 ; \mathrm{fn} 1 \mathrm{~b}=$ fibronectin $1 \mathrm{~b}$; aldh $1 \mathrm{a} 2$ = aldehyde dehydrogenase 1 family, member $\mathrm{A} 2$; ctsd = cathepsin $\mathrm{D}$; ugt $1 \mathrm{ab}=$ UDP-glucuronosyltransferase 1ab; cyp3a65 = cytochrome P450 3a65. 


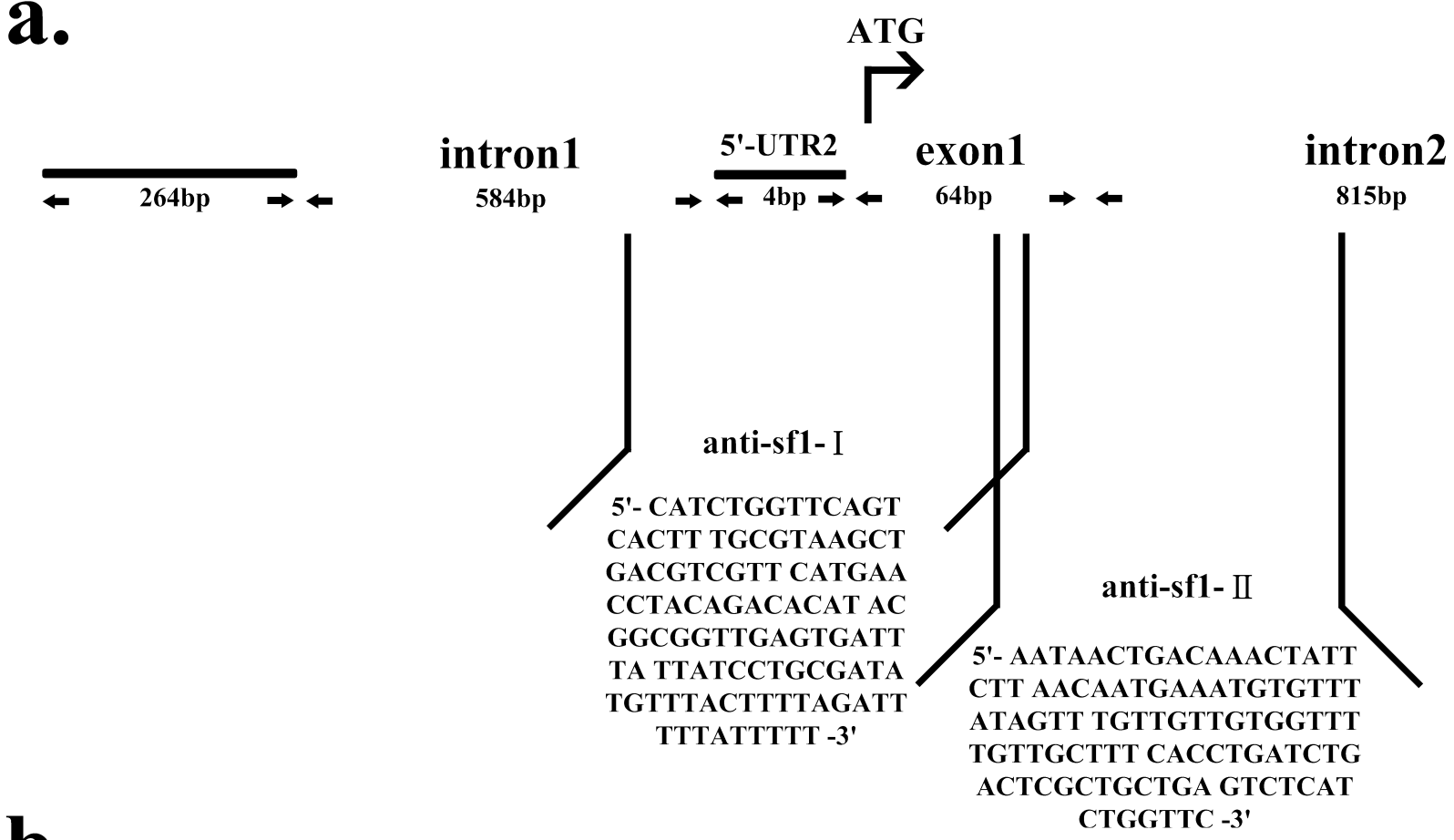

b.

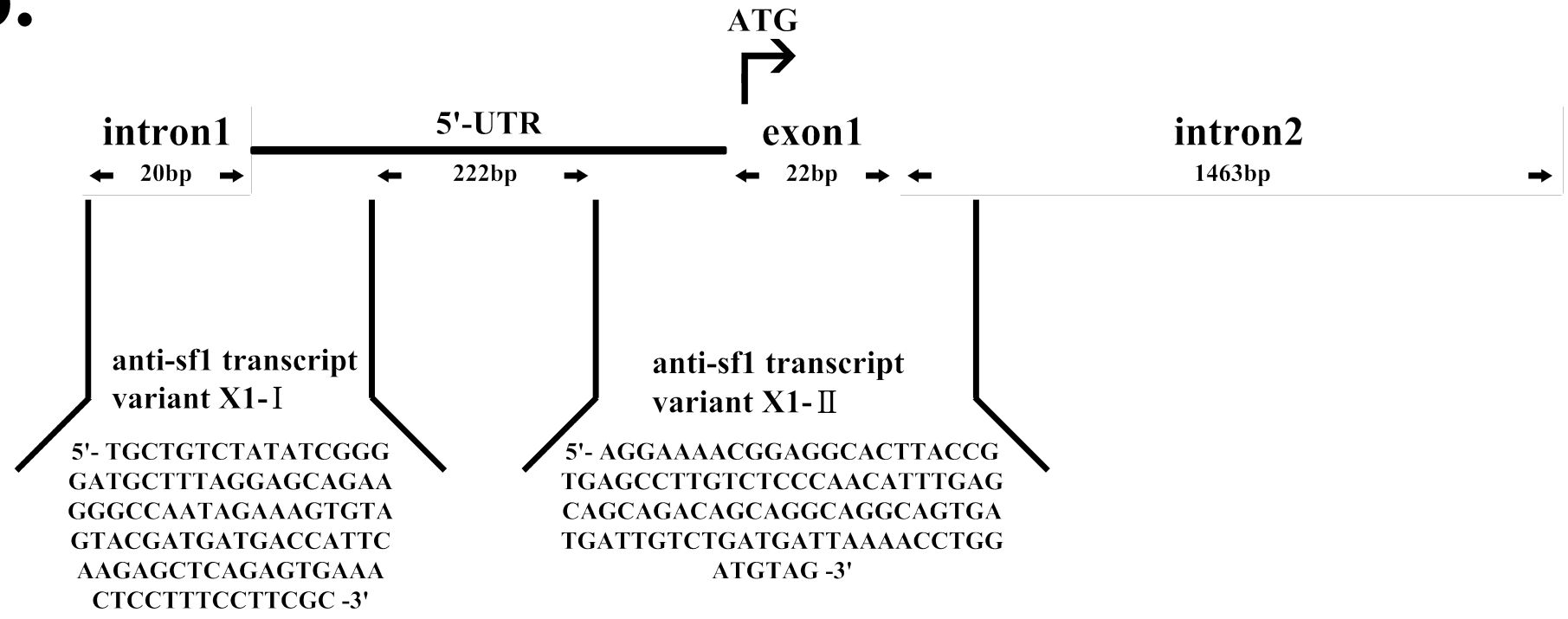

Figure 5

Design and action site of antisense RNA sequence of sf1. a Sequence information and interference sites

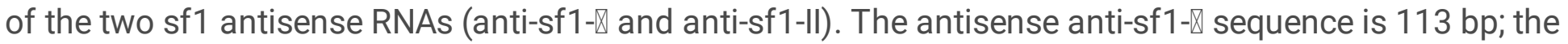
interference site contains the antisense sequence of a first intron partial sequence, a first exon partial sequence, and a 5'- non-translated sequence of four bases of the sf 1 mRNA precursor. The antisense antisf1-II sequence is $104 \mathrm{bp}$; the interference site contains the antisense sequence of a second intron partial sequence, and a first exon partial sequence of the sf1 mRNA precursor. b Sequence information and

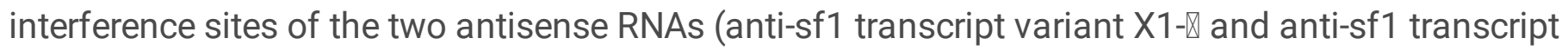
variant $X 1-\nabla)$ of sf1 transcript variant $X 1$. The antisense RNA sequence of the anti-sf1 transcript variant $\mathrm{X} 1-\mathbb{Z}$ is $102 \mathrm{bp}$ in size; the interference site contains the antisense sequence of a first intron partial 
sequence, and a 5'-non-translated sequence of 80 bases of sf 1 transcript variant X1 mRNA precursor. The antisense RNA sequence of the anti-sf1 transcript variant X1- $\$ is $102 \mathrm{bp}$ in size, and the interference site contains the antisense sequence of a second intron partial sequence, a first exon sequence, and a $5^{\prime}$-non translated sequence of 60 bases of sf 1 transcript variant $\mathrm{X} 1 \mathrm{mRNA}$ precursor.

\section{Supplementary Files}

This is a list of supplementary files associated with this preprint. Click to download.

- SupplementaryTable.docx

- SupplementaryFigs.docx

- SourceDatafemale.xlsx

- SourceDatamale.xlsx 